\title{
Breast milk protects against the development of necrotizing enterocolitis through inhibition of Toll-like receptor 4 in the intestinal epithelium via activation of the epidermal growth factor receptor
}

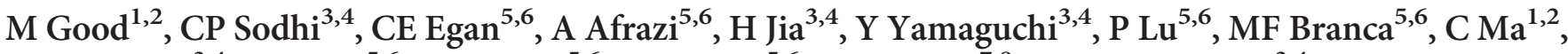 \\ T Prindle Jr ${ }^{3,4}$, S Mielo ${ }^{5,6}$, A Pompa ${ }^{5,6}$, Z Hodzic ${ }^{5,6}$, JA Ozolek ${ }^{7,8}$ and DJ Hackam ${ }^{3,4}$
}

Breast milk is the most effective strategy to protect infants against necrotizing enterocolitis (NEC), a devastating disease that is characterized by severe intestinal necrosis. Previous studies have demonstrated that the lipopolysaccharide receptor Toll-like receptor 4 (TLR4) plays a critical role in NEC development via deleterious effects on mucosal injury and repair. We now hypothesize that breast milk protects against NEC by inhibiting TLR4 within the intestinal epithelium, and sought to determine the mechanisms involved. Breast milk protected against NEC and reduced TLR4 signaling in wildtype neonatal mice, but not in mice lacking the epidermal growth factor receptor (EGFR), whereas selective removal of EGF from breast milk reduced its protective properties, indicating that breast milk inhibits NEC and attenuates TLR4 signaling via EGF/EGFR activation. Overexpression of TLR4 in the intestinal epithelium reversed the protective effects of breast milk. The protective effects of breast milk occurred via inhibition of enterocyte apoptosis and restoration of enterocyte proliferation. Importantly, in IEC-6 enterocytes, breast milk inhibited TLR4 signaling via inhibition of glycogen

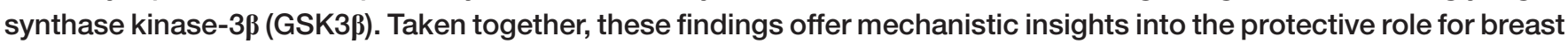
milk in NEC, and support a link between growth factor and innate immune receptors in NEC pathogenesis.

\section{INTRODUCTION}

Necrotizing enterocolitis (NEC) is the most common and lethal gastrointestinal emergency in preterm infants, ${ }^{1}$ and despite extensive research investigating the underpinnings of this disease, the molecular mechanisms involved remain incompletely understood. NEC is characterized by the acute onset of patchy intestinal necrosis and systemic sepsis, and is seen most commonly in premature infants, of whom $\sim 12 \%$ will develop this devastating disease. ${ }^{1,2}$ Studies from several labs including our own have shown that the development of NEC reflects the interaction between the bacteria that inhabit the intestinal tract of the premature infant and the underlying intestinal mucosa, leading to a marked pro-inflammatory response that requires activation of the lipopolysaccharide receptor Toll-like receptor 4 (TLR4) on the intestinal epithelium. ${ }^{3-5}$ Activation of TLR4 leads to increased enterocyte apoptosis and reduced mucosal healing, ${ }^{6,7}$ and also contributes to the impaired intestinal perfusion that leads to intestinal necrosis that is a cardinal feature of NEC. ${ }^{8}$ The importance of intestinal TLR4 in the pathogenesis of NEC is revealed by studies in which mice deficient in TLR4 in the intestinal epithelium are protected from NEC development, ${ }^{4}$ as are wild-type mice that have received a novel TLR4 inhibitor. ${ }^{9}$ It has been long recognized that breast milk is an effective protective agent against NEC, ${ }^{10-13}$

\footnotetext{
${ }^{1}$ Division of Newborn Medicine, Children's Hospital of Pittsburgh, Pittsburgh, Pennsylvania, USA. ²Department of Pediatrics, University of Pittsburgh School of Medicine, Pittsburgh, Pennsylvania, USA. ${ }^{3}$ Division of General Pediatric Surgery, Johns Hopkins University and Bloomberg Children's Center, Johns Hopkins Hospital, Baltimore, Maryland, USA. ${ }^{4}$ Department of Surgery, Johns Hopkins University, Baltimore, Maryland, USA. 'Division of Pediatric Surgery, Children's Hospital of Pittsburgh, Pittsburgh, Pennsylvania, USA. ${ }^{6}$ Department of Surgery, University of Pittsburgh School of Medicine, Pittsburgh, Pennsylvania, USA. ${ }^{7}$ Division of Pediatric Pathology, Children's Hospital of Pittsburgh, Pittsburgh, Pennsylvania, USA and ${ }^{8}$ Department of Pathology, University of Pittsburgh School of Medicine, Pittsburgh, Pennsylvania, USA. Correspondence: DJ Hackam (dhackam1@jhmi.edu)
} 
although the components in breast milk that mediate this protection and the signaling pathways involved remain incompletely understood. Epidermal growth factor (EGF) is abundant in breast milk and amniotic fluid and is critical for intestinal development. ${ }^{14-18}$ Our laboratory has shown that amniotic fluid inhibits TLR4 signaling in the neonatal intestinal epithelium via the epidermal growth factor receptor (EGFR). ${ }^{19}$ In view of these findings and the growing literature on the causative role for intestinal TLR4 in the pathogenesis of NEC from our lab ${ }^{3,4,19-21}$ and others, ${ }^{5,22,23}$ we now hypothesize that breast milk prevents NEC via inhibition of TLR4 signaling, and sought to define the mechanisms involved. In support of this hypothesis, we now show that breast milk prevents the exaggerated TLR4 signaling that is required for NEC via effects on the EGFR.

\section{RESULTS}

\section{Breast milk inhibits TLR4 signaling in enterocytes via} activation of the EGFR

We first sought to determine whether breast milk could inhibit TLR4 signaling in enterocytes in vitro. To do so, we treated IEC6 enterocytes, which we and others have previously shown to express both TLR4 (see refs. 3,19,24-26) and EGFR, ${ }^{19}$ with lipopolysaccharide (LPS), and the degree of TLR4 signaling was measured by assessing the extent of translocation of the p65 subunit of nuclear factor (NF)- $\mathrm{BB}$ from the cytoplasm to the nucleus and the expression of the pro-inflammatory cytokine interleukin-6 (IL-6). Consistent with our previous observations, ${ }^{19}$ LPS induced TLR4 signaling in IEC-6 cells, as shown in Figure 1. Pretreatment of cells with breast milk significantly reduced the extent of LPS-induced TLR4-mediated nuclear translocation of NF- $\kappa B$ (Figure 1aii, iii, ei), IL-6 induction (Figure 1eii), and TLR4 expression (Figure 1eiii). The protective effects of breast milk on TLR4 signaling could be reversed after preheating the milk to $95^{\circ} \mathrm{C}$, suggesting that a heat-sensitive factor, likely a protein, was involved in the downregulatory effect of breast milk on TLR4 (Figure 1avi, ei). Given our previous studies that indicated that EGFR activation by components of amniotic fluid could inhibit TLR4 signaling, ${ }^{19}$ we explored whether EGFR was required for the breast milk-mediated inhibition of TLR4. In support of this possibility, breast milk did not prevent LPS-mediated TLR4 signaling in IEC-6 cells that had undergone lentiviral-mediated knockdown of EGFR (Figure 1bii, iii, d, fi, ii). Furthermore, immunodepletion of EGF from breast milk reversed the inhibitory effects of breast milk on TLR4 signaling (Figure 1aiv vs. iii, ei), whereas replenishment of EGF-depleted breast milk with EGF restored its ability to inhibit TLR4 signaling in wildtype enterocytes (Figure 1av vs. iv, ei), but not in EGFRdeficient enterocytes (Figure 1 biii-v, fi). Taken together, these findings indicate that breast milk inhibits TLR4 signaling via activation of the EGF receptor by EGF.

\section{Breast milk attenuates TLR4 signaling in vivo via EGFR}

We next sought to determine whether breast milk could also inhibit TLR4 signaling in vivo, and thus potentially explain its protective effects in NEC. To determine the degree that breast milk inhibits LPS-mediated TLR4 signaling, we administered breast milk by oral gavage to transgenic neonatal mice that express NF- $\kappa \mathrm{B}$ on the luciferase promoter. At $1 \mathrm{~h}$ after oral gavage with either saline (Figure 2ai, v) or breast milk (Figure 2aiii, v), the NF-кB-luciferase-expressing mice were injected with LPS in order to induce TLR4 activation. After luciferin injection, whole-animal imaging was performed to assess NF- $\kappa \mathrm{B}$ activity as determined by the extent of luciferase activity. ${ }^{9}$ As shown in Figure 2, injection of mice with LPS caused a significant increase in whole-animal luciferase activity, whereas animals that were orally administered breast milk $1 \mathrm{~h}$ before LPS injection demonstrated significantly decreased luciferase (i.e., NF- $\kappa \mathrm{B}$ ) activity along with decreased expression of the pro-inflammatory cytokine IL- 6 and TLR4 within the intestine (Figure 2aii, iii, v, bi, ii). In order to evaluate whether EGFR activation was required for the inhibition of TLR4 signaling in the intestine in vivo, we performed experiments in the presence of the selective EGFR inhibitor cetuximab. As shown in Figure 2aiv, pretreatment with cetuximab prevented the protection of breast milk on TLR4 signaling, as shown by a lack of inhibitory effect of breast milk on NF- $\mathrm{KB}$ luciferase activity (Figure 2aiv vs. iii, v, bi, ii). Taken together, these findings support the conclusion that breast milk inhibits TLR4 in vivo via EGFR activation.

\section{Breast milk reversed TLR4-mediated induction of apoptosis in the intestinal epithelium in an EGFR-dependent manner}

We and others have shown that TLR4 activation induces intestinal epithelial injury in NEC in part through induction of enterocyte apoptosis. ${ }^{6,7,19-21,27,28}$ Having shown that breast milk inhibits TLR4 signaling in vivo, we next sought to evaluate the effects of breast milk on TLR4-induced inhibition of proliferation and induction of apoptosis, and whether EGFR signaling could be involved. As shown in Figure 3, injection of LPS into newborn pups caused a marked increase in apoptosis of enterocytes as revealed by increased TUNEL (terminal deoxynucleotidyl transferase dUTP nick end labeling)-positive cells in the intestinal epithelium (Figure 3ai, ii, arrows, di) as well as increased 3-nitrotyrosine staining that is a marker of gut inflammation seen in NEC, ${ }^{29,30}$ known to be TLR4 dependent ${ }^{31}$ and is a by-product of nitric oxide signaling derived from peroxynitrite $^{29}$, which were reversed by gavage pretreatment with breast milk (Figure 3aii, iii, di). Three lines of evidence suggest that EGFR activation by EGF is responsible for the protective effects of breast milk on enterocyte apoptosis and proliferation. First, pretreatment of mice with the EGFR inhibitor cetuximab reversed the protective effects of breast milk on TLR4-mediated inhibition of proliferation and induction of apoptosis (Figure 3aiv vs. iii, di). Second, immunodepletion of EGF from breast milk reversed the protective effects of breast milk on apoptosis (Figure 3av vs. iii, di), whereas reconstitution of breast milk with EGF restored the protective properties of breast milk (Figure 3avi vs. v, di). Third, mice lacking EGFR in the intestinal epithelium 

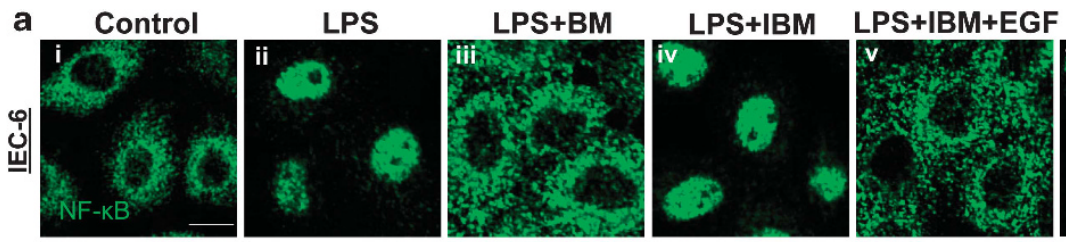

LPS+BBM
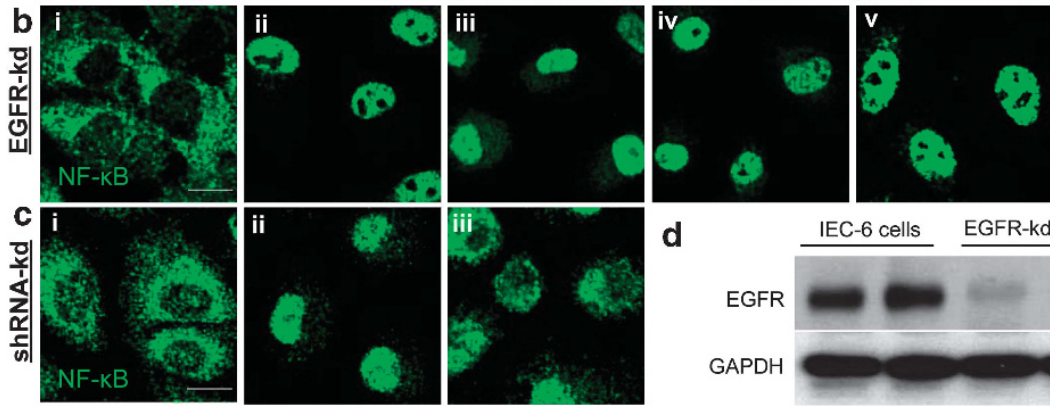

d

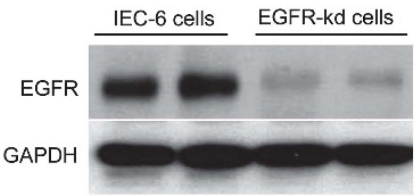

e
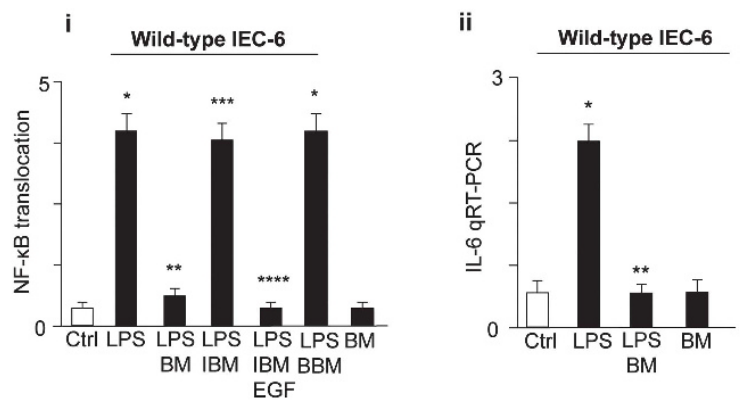

iii Wild-type IEC-6
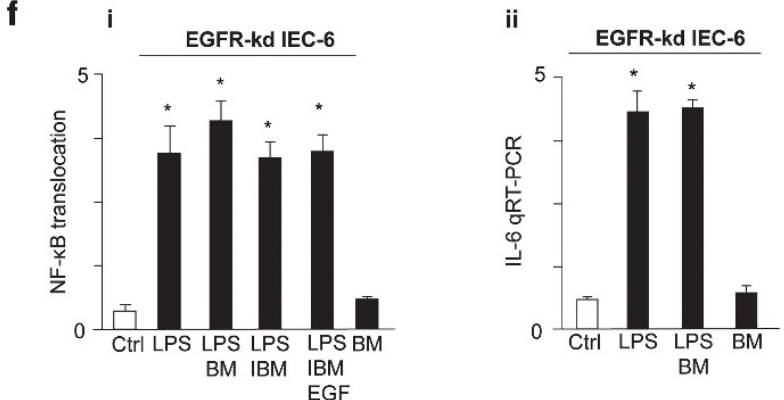

Figure 1 Breast milk (BM) inhibits Toll-like receptor 4 (TLR4) signaling in enterocytes via activation of the epidermal growth factor receptor (EGFR) by EGF. (a-c) Representative confocal micrographs of either wild-type IEC-6 cells (ai-vi) or IEC-6 cells that were deficient in EGFR (bi-v) or control (Ctrl) scrambled short hairpin (shRNA; ci-iii) that were treated with either lipopolysaccharide (LPS; $50 \mu \mathrm{g} \mathrm{ml}^{-1}, 1 \mathrm{~h}$, aii, bii, cii) and after $1 \mathrm{~h}$ of pretreatment with breast milk $\left(25 \mu \mathrm{lml} \mathrm{m}^{-1}\right.$ media, aiii, biii, ciii), or EGF-immunodepleted breast milk (IBM, aiv, biv), IBM with exogenous EGF $\left(400 \mathrm{ng} \mathrm{ml}^{-1}\right.$ media, av, bv) or boiled breast milk (BBM). Scale bar $=10 \mu \mathrm{m}$. The p65 subunit of nuclear factor (NF)- $\kappa B$ (green). (d) Real-time PCR (RT-PCR) showing EGFR (upper) and loading control glyceraldehyde 3-phosphate dehydrogenase (GAPDH; lower) in wild-type (left lanes), EGFR knockdown (EGFR-kd) IEC-6 cells (right lanes). (e) Quantification of the extent of NF-kB translocation (ei). Interleukin-6 (IL-6) mRNA expression (LPS $50 \mu \mathrm{g} \mathrm{ml}^{-1}, 6 \mathrm{~h}$, eii) or TLR4 mRNA expression (LPS, $50 \mu \mathrm{g} \mathrm{ml}^{-1}, 24 \mathrm{~h}$, eiii) and the above pretreatments with breast milk in wild-type IEC-6 cells. (f) NF- $\kappa B$ translocation under the above conditions in EGFR-kd IEC-6 cells (fi) and IL-6 mRNA expression (fii). ${ }^{*} P<0.05$ vs. control (white bars), ${ }^{* \star} P<0.05$ vs. LPS, ${ }^{* * \star} P<0.05$ vs. LPS + BM by analysis of variance (ANOVA). These data are mean \pm s.d. Results are representative of at least three separate experiments with $>50$ fields each. Scale bar $=10 \mu \mathrm{m}$.

$\left(\mathrm{EGFR}^{\mathrm{\Delta IEC}}\right.$ mice) were found to demonstrate susceptibility to LPS-induced apoptosis that was not protected by the presence of breast milk (Figure $\mathbf{3 b i - i v , ~ d i i ) . ~ I t ~ i s ~ a l s o ~ n o t e w o r t h y ~ t h a t ~}$ TLR $4^{\Delta \text { IEC-OVER }}$ mice, in which TLR4 was overexpressed in the intestinal epithelium on a global TLR4 KO background, demonstrated increased enterocyte apoptosis that was not protected by breast milk (Figure 3ci-iv, diii), indicating that TLR4 signaling when maintained at high levels overcomes the protective effects of breast milk. Taken together, these findings illustrate that LPS-mediated TLR4 signaling in the intestinal mucosa is attenuated by breast milk through activation of EGFR. We next sought to determine the mechanisms involved.

\section{Breast milk restores enterocyte proliferation via EGF and the EGFR}

Previously, we have shown that TLR4 activation inhibits enterocyte proliferation in experimental models of endotoxemia and NEC. ${ }^{6,7,19-21,27}$ As shown in Figure 4, in wild-type 

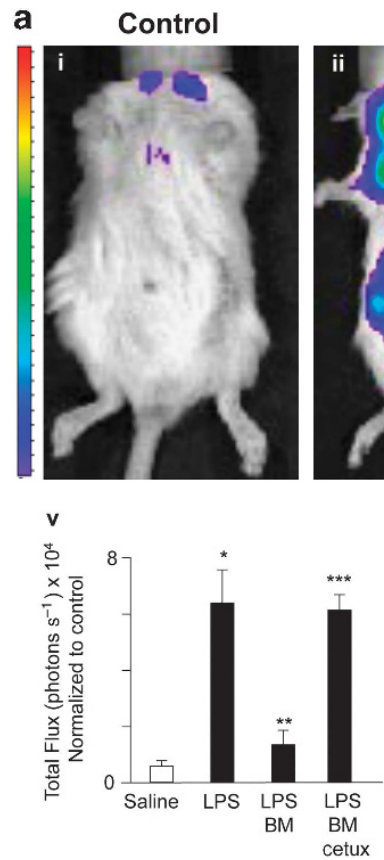

LPS

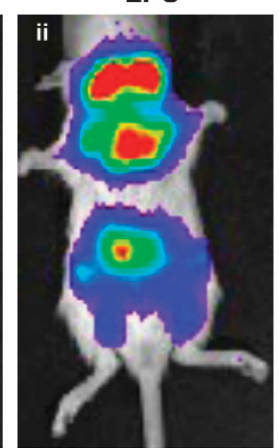

b i

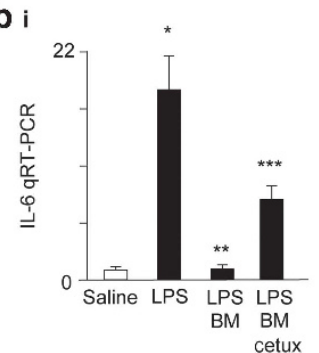

LPS+BM

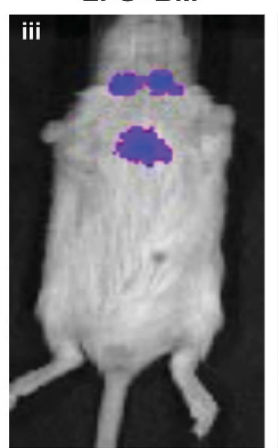

ii

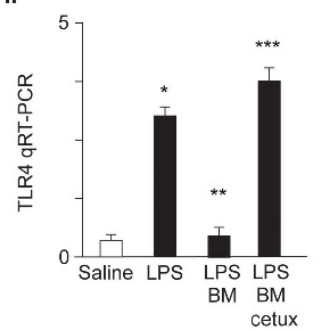

Figure 2 Breast milk (BM) attenuates Toll-like receptor 4 (TLR4) signaling in vivo via epidermal growth factor receptor (EGFR). (a) Pseudocolor images demonstrating whole-animal nuclear factor (NF)-кB luciferase activity treated with either saline (i), lipopolysaccharide (LPS; $\left.5 \mathrm{mg} \mathrm{kg}{ }^{-1}, 6 \mathrm{~h}, \mathrm{ii}\right)$, gavage pretreatment with breast milk ( $50 \mu \mathrm{l}$ per g body weight, iii), and/or cetuximab (Cetux), an EGFR inhibitor (100 $\mu \mathrm{g}$ per day for 3 days, iv) $1 \mathrm{~h}$ before LPS administration, and quantification of images in Total Flux (photons $\mathrm{s}^{-1} \times 10^{4}$, v). (b) Interleukin-6 (IL-6) (i) and TLR4 mRNA expression (ii) for the above groups. ${ }^{\star} P<0.05$ vs. saline (white bar), ${ }^{\star \star} P<0.05$ vs. LPS, ${ }^{* * *} P<0.05$ vs. LPS + BM. Data are mean \pm s.d. Results are representative of at least three separate experiments with at least three mice per group.

mice LPS inhibited enterocyte proliferation as revealed by a reduction in proliferating cell nuclear antigen staining that was reversed by pretreatment with breast milk (Figure 4ai-iii, ci). Pretreatment of mice with the EGFR inhibitor cetuximab (Figure 4aiv) or immunodepletion of EGF from breast milk (Figure 4av) reversed the protective effects of breast milk on enterocyte proliferation, and this was restored in the presence of EGF-supplemented breast milk (Figures 4avi, ci). As shown in Figure 4, EGFR ${ }^{\Delta I E C}$ mice were also found to demonstrate a LPS-mediated inhibition of proliferation that was not restored in the presence of breast milk treatment (Figure 4bi-iii, cii). These findings provide additional support for the role of EGF and EGFR in the protection against TLR4-mediated signaling by breast milk in the neonatal gut.

\section{Breast milk attenuates TLR4-mediated NF-кB activation by inhibiting GSK3 $\beta$ in vitro}

The glycogen synthase kinase-3 $\beta$ (GSK3 $\beta$ )/ $\beta$-catenin signaling pathway plays a key role in determining the extent of enterocyte proliferation that occurs in response to EGFR ligands and is situated canonically downstream of both EGFR and TLR4. ${ }^{32-35}$ To assess the potential mechanisms by which EGFR activation by breast milk inhibits TLR4 in the intestinal epithelium, we next explored whether impaired GSK3 $\beta$ signaling may be involved. As shown in Figure 5, in wild-type IEC-6 cells, pretreatment with either EGF or breast milk before LPS administration significantly increased the degree of phosphorylation of GSK3 $\beta$ at serine 9 as determined by sodium dodecyl sulfate-polyacrylamide gel electrophoresis (SDS-PAGE; Figure 5ai, ii) and confocal microscopy (Figure 5b). Given that phosphorylation of GSK $3 \beta$ is associated with its inactivation, ${ }^{34-36}$ these findings suggest that GSK3 $\beta$ inhibition may underlie the effects of breast milk on TLR4. To evaluate this possibility directly, we inhibited or inactivated GSK3 $\beta$ in enterocytes and assessed whether breast milk was still capable of exerting its negative effects on TLR4 signaling. To do so, we first treated cells with the GSK3 $\beta$ inhibitor lithium chloride that blocks GSK3 $\beta$ signaling via phosphorylation of serine $9,{ }^{37,38}$ and by phosphorylating the $\mathrm{N}$-terminal serine residues of GSK $3 \beta .^{39,40}$ As shown in Figure 5ai, ii, pretreatment with lithium chloride increased the phosphorylation of GSK3 $\beta$ and also decreased the expression of TLR4mediated IL-1 $\beta$ (Figure 5aiii) and IL-6 (Figure 5aiv) in IEC-6 cells. In parallel, we knocked down GSK3 $\beta$ via lentiviral transduction of GSK3 $\beta$ short hairpin RNA (Figure 5civ). As shown in Figure 5c, treatment of GSK3 $\beta$-kd cells with LPS significantly increased $\mathrm{NF}-\kappa \mathrm{B}$ translocation and the proinflammatory cytokine IL-6 (Figure 5Ci, ii, v, vi), similar to the situation in wild-type cells (Figure 1ai, ii, ei, ii) that was not reversed by breast milk (Figure 5ciii, v, vi). Taken together, these findings strongly imply that breast milk inhibits TLR4 activation in enterocytes through the effects of GSK3 $\beta$ signaling.

\section{Breast milk inhibits TLR4-mediated apoptosis and proliferation in enterocytes via EGFR and GSK3ß.}

Shown in Figures 6 and 7 is evidence that breast milk reversed the effects of TLR4 on apoptosis and proliferation via EGFR 
a
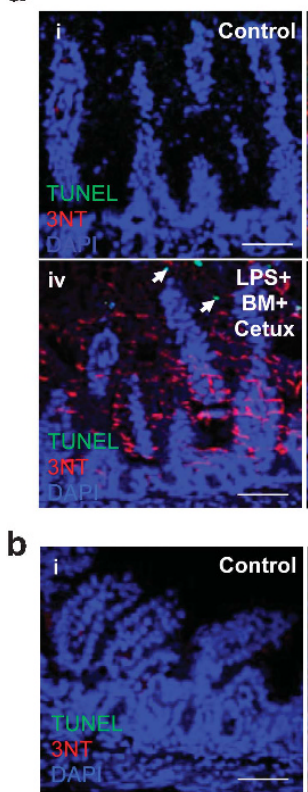

c

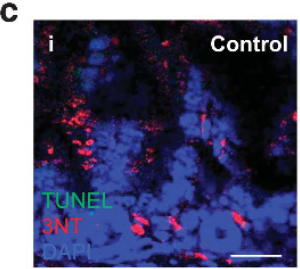

d

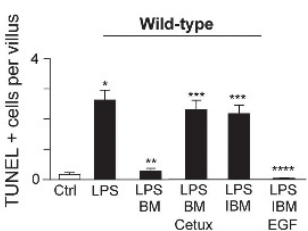

Wild-type

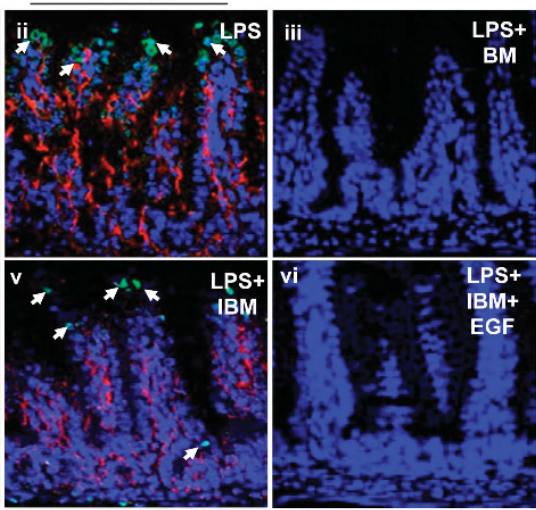

$E$ GFR $^{\triangle I E C}$
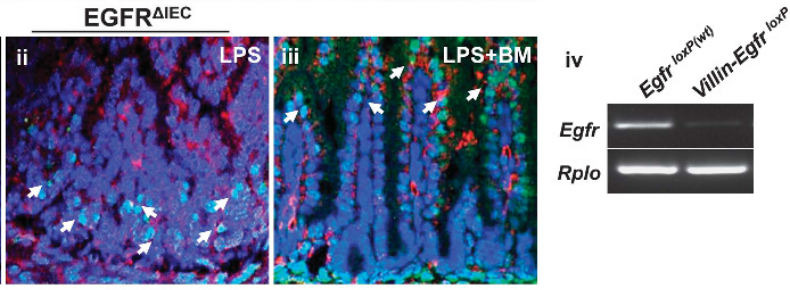

TLR4 $\triangle$ IEC-OVER
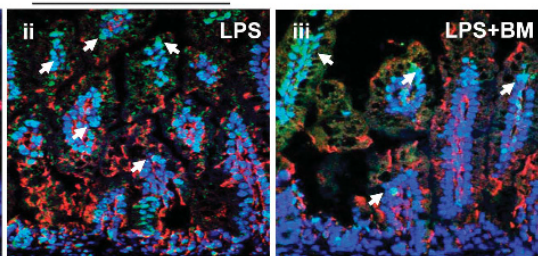

iv

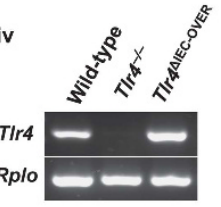

Figure 3 Breast milk (BM) reversed Toll-like receptor 4 (TLR4)-mediated induction of apoptosis in the intestinal epithelium in an epidermal growth factor receptor (EGFR)-dependent manner. (a-c) Representative confocal micrographs of the terminal ileum of either wild-type mice (ai-vi), EGFR ${ }^{\Delta I E C}$ mice (bi-iv), or TLR4 ${ }^{\mathrm{LIEC}-\mathrm{OVER}}$ mice (ci-iv) stained for terminal deoxynucleotidyl transferase dUTP nick end labeling (TUNEL; green, arrows), 3-nitrotyrosine (3NT, red), and 4',6-diamidino-2-phenylindole (DAPI; blue) as indicated after treatments with saline (Control (Ctrl), ai, bi, ci), lipopolysaccharide (LPS; $5 \mathrm{mg} \mathrm{kg}^{-1}, 16 \mathrm{~h}$, aii, bii; $2.5 \mathrm{mg} \mathrm{kg}^{-1}, 6 \mathrm{~h}$, cii), LPS + breast milk (LPS + BM, aiii, biii, ciii), LPS + breast milk + cetuximab (LPS + BM + Cetux, aiv), LPS + EGF- immunodepleted breast milk (LPS + IBM, av), LPS + IBM + EGF (avi). Scale bars $=100 \mu \mathrm{m}$. Real-time PCR (RT-PCR) showing expression of EGFR (biv, upper) or TLR4 (civ, upper) within the small intestine of the indicated strains, along with the housekeeping gene RPLO (biv, civ, lower). (d) Quantification of the TUNEL-positive cells per villus in wild-type mice (i), EGFR ${ }^{\Delta \mathrm{IEC}}$ mice (ii), or TLR ${ }^{\Delta \mathrm{IEC}-\mathrm{OVER}}$ mice (iii). ${ }^{\star} P<0.05$ vs. saline (Ctrl, white bar), ${ }^{\star \star} P<0.05$ vs. LPS, ${ }^{\star \star \star} P<0.05$ vs. LPS + BM. ${ }^{\star \star * \star} P<0.05$ vs. LPS + IBM. Data are mean \pm s.e.m. Results are representative of at least three separate experiments with at least three mice per group. Arrows delineate apoptotic cells. Scale bar $=100 \mu \mathrm{m}$.

activation by EGF in a GSK3 $\beta$-dependent manner. In wild-type cells, breast milk protected against TLR4-mediated enterocyte apoptosis (Figure 6aiii vs. ii, di) and increased enterocyte proliferation (Figure 7aiii vs. ii, di), whereas immunodepletion of EGF from breast milk blocked the protective effects on TLR4 signaling (Figures 6aiv vs. iii, di and 7aiv vs. iii, di) that was restored by replacement with exogenous EGF (Figure 6av vs. iv, di and 7 av vs. iv, di). In parallel, breast milk treatment of IEC-6 cells in which EGFR had been knocked down were not protected from TLR4-mediated apoptosis (Figure $6 \mathbf{b i}-\mathbf{v}$, dii) or proliferation (Figure $7 \mathbf{b i}-\mathbf{v}$, dii). Similarly, treatment with breast milk in GSK3 $\beta$-deficient cells did not protect against apoptosis (Figure 6ci-iii, diii) or proliferation (Figure 7ci-iii, diii). Taken together, these findings suggest that breast milk reverses the effects of TLR4 on enterocyte apoptosis and proliferation via EGFR and its inhibition of GSK3 $\beta$.

\section{Breast milk attenuates NEC severity via EGFR activation}

In the next series of studies, we sought to investigate whether breast milk could protect against the development of NEC through EGFR activation. As shown in Figures 8 and 9, experimental NEC was induced in newborn pups using an established model that involves a combination of formula 
a

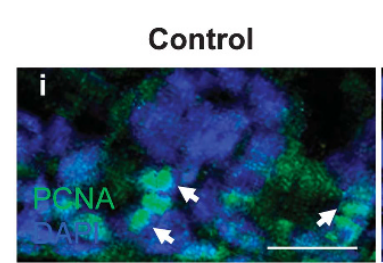

LPS+BM+Cetux
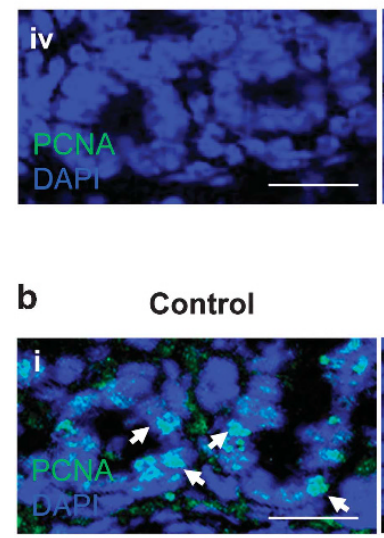

c

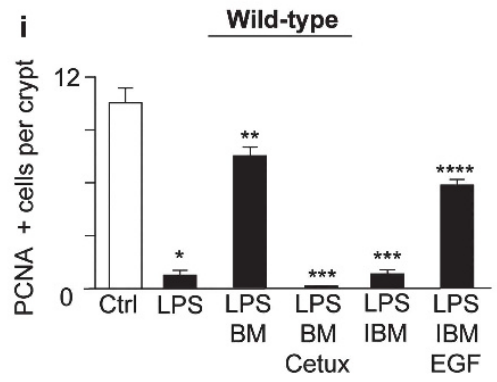

Wild-type

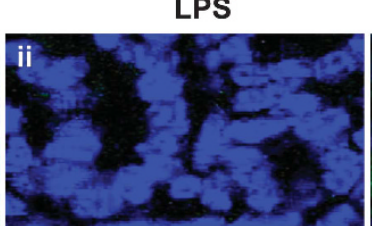

LPS+IBM

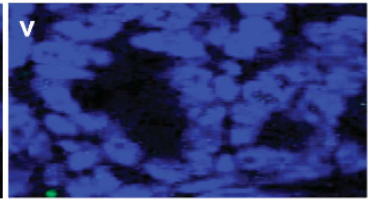

$E^{E G F R}{ }^{\Delta I E C}$

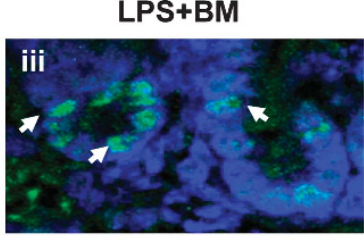

LPS+IBM+EGF

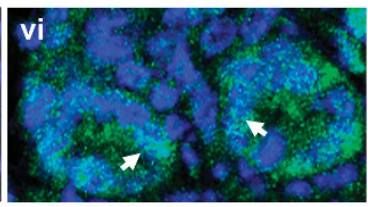

LPS+BM
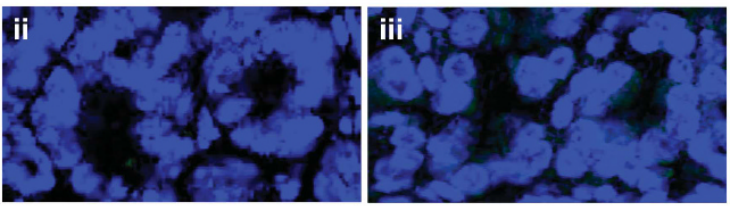

ii

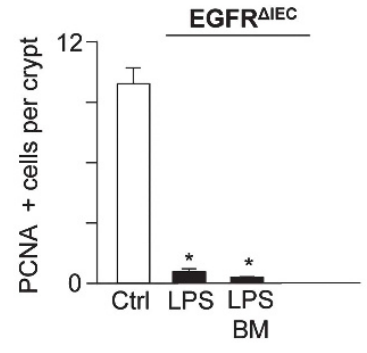

Figure 4 Breast milk (BM) restores enterocyte proliferation via epidermal growth factor (EGF) and the EGF receptor (EGFR). (a, b) Representative confocal micrographs of the small intestinal crypts of either wild-type mice (ai-vi) or EGFR ${ }^{\Delta I E C}$ mice (bi-iii) stained for proliferating cell nuclear antigen (PCNA; green, arrows) and 4',6-diamidino-2-phenylindole (DAPI; blue) as indicated after treatments with saline (Control (Ctrl), ai, bi), lipopolysaccharide (LPS; $5 \mathrm{mg} \mathrm{kg}^{-1}, 6 \mathrm{~h}$, aii, bii), LPS + breast milk (LPS + BM, aiii, biii), LPS + breast milk + cetuximab (LPS + BM + Cetux, aiv), LPS + EGF-

immunodepleted breast milk (LPS + IBM, av), and LPS + IBM + EGF (avi). Scale bars $=50 \mu \mathrm{m}$. (c) Quantification of the PCNA-positive cells per crypt in wild-type mice (i) or EGFR ${ }^{\Delta l E C}$ mice (ii). ${ }^{\star} P<0.05$ vs. saline (Ctrl, white bar), ${ }^{\star \star} P<0.05$ vs. LPS, ${ }^{\star \star \star} P<0.05$ vs. LPS $+B M$, and ${ }^{\star \star * \star} P<0.05$ vs. LPS + IBM. Data are mean \pm s.e.m. Results are representative of at least three separate experiments with at least three mice per group. Arrows indicate proliferative cells. Scale bar $=50 \mu \mathrm{m}$.

gavage and hypoxia treatment. ${ }^{4,4,41,42}$ The administration of breast milk significantly attenuated experimental NEC, as manifest by a reduction in NEC severity (Figure 8ai, ii, cii), the pro-inflammatory gene expression inducible nitric oxide synthase (Figure 8ci), as well as a reduction in enterocyte apoptosis and 3-nitrotyrosine (Figure 9ai-iii, ei), and enhanced proliferation (Figure 9bi-iii, fi). Importantly, mice lacking intestinal EGFR were found to exhibit increased NEC severity compared with wild-type mice that was not reduced by the administration of breast milk (Figure 8bi-iii, di, ii). Breast milk did not protect against NEC-mediated enterocyte apoptosis (Figure 9ci-iii, eii) nor enhance enterocyte proliferation (Figure 9di-iii, fii) in $\mathrm{EGFR}^{\Delta \mathrm{IEC}}$ mice, indicating that breast milk activation of EGFR is required for these protective effects. In addition, pretreatment of animals with cetuximab in conjunction with breast milk blocked the protective effects of breast milk on NEC severity (Figure 8aiv vs. iii, ci, ii), enterocyte apoptosis (Figure 9aiv vs. iii, ei), and proliferation (Figure 9biv vs. iii, fi). Wild-type animals that were treated with the EGF-depleted milk had loss of the protective effects of breast milk on NEC severity (Figure 8av, cii), concomitant with expression of inducible nitric oxide synthase (Figure 8ci), enterocyte apoptosis (Figure 9av, ei), and reduced proliferation (Figure 9bv, fi), all of which were restored when recombinant EGF was supplemented (Figures 8avi, ci, cii and 9avi, bvi, ei, fi). Taken together, these findings indicate that breast milk protects against experimental NEC in part through inhibition of TLR4 and that this protective effect requires EGF/EGFR signaling. 
a
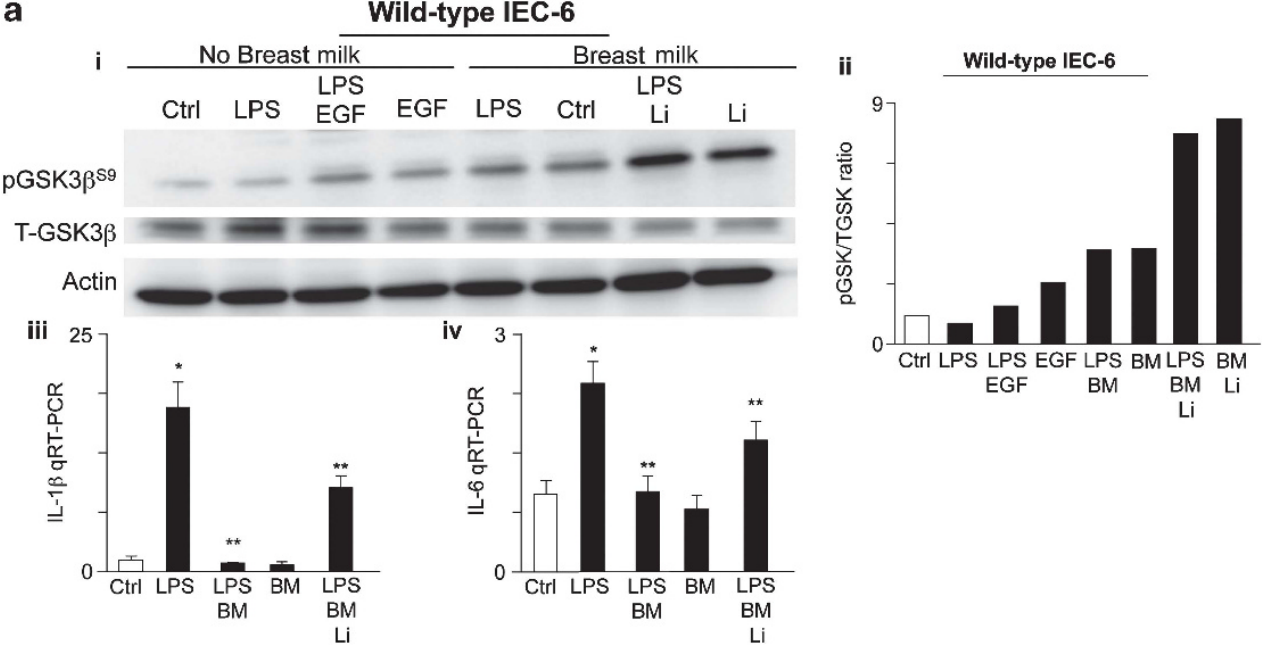

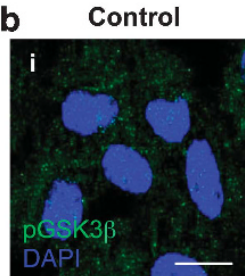

c

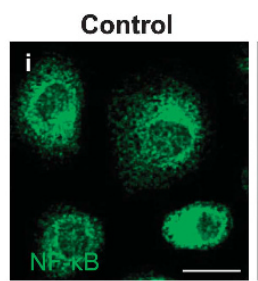

iv

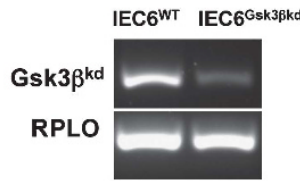

LPS

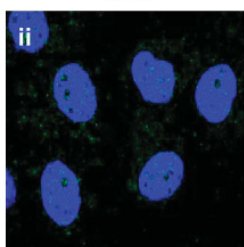

GSK3 $\beta$-kd IEC-6
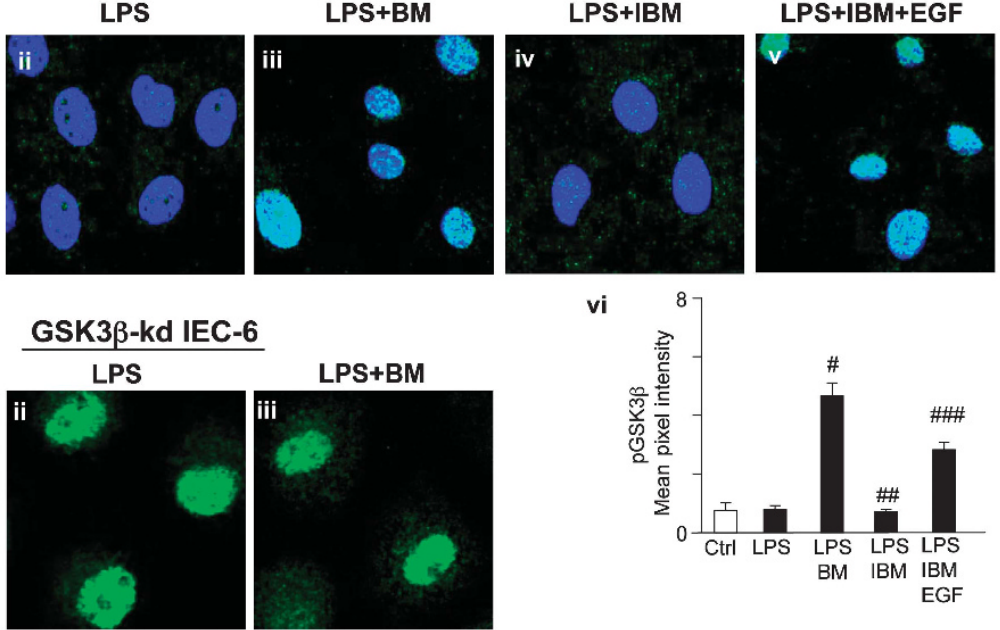

vi
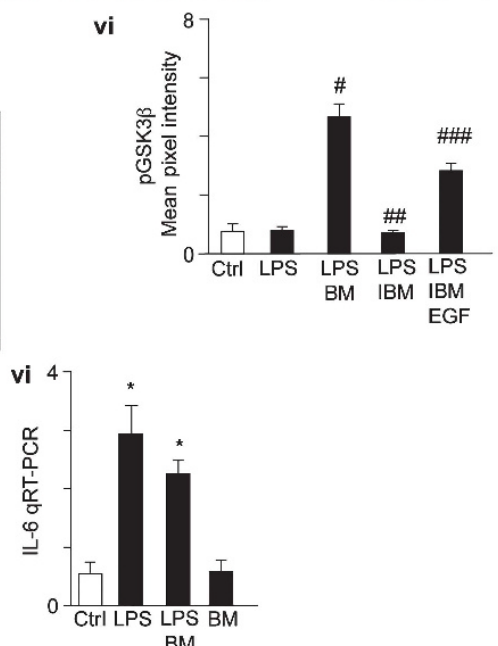

Figure 5 Breast milk (BM) attenuates Toll-like receptor 4 (TLR4)-mediated nuclear factor (NF)- $\kappa B$ activation by inhibiting glycogen synthase kinase-3 $\beta$ (GSK3 $\beta$ ) in vitro. (a) Sodium dodecyl sulfate-polyacrylamide gel electrophoresis (SDS-PAGE) showing the phosphorylation of GSK3 $\beta$ at serine 9 (pGSK3 $\beta^{\text {S9 }}$, upper), total GSK3 $\beta$ (T-GSK3 $\beta$, middle), and actin (loading control, lower) in serum- and insulin-starved wild-type IEC-6 cells, with treatment groups as indicated (lipopolysaccharide (LPS) $50 \mu \mathrm{g} \mathrm{ml}^{-1}, 15 \mathrm{~min}$, after $1 \mathrm{~h}$ pretreatment with epidermal growth factor (EGF) $400 \mathrm{ng} \mathrm{ml}{ }^{-1}$ or breast milk $50 \mu \mathrm{lml} \mathrm{m}^{-1}$ media or lithium chloride (Li), $100 \mu \mathrm{M}$, i); blots were stripped and reprobed for T-GSK3 $\beta$ and actin. Quantification of pGSK3 $\beta$ to total GSK3 $\beta$ expression ratio (ImageJ Software, NIH, ii). Interleukin (IL)-1 $\beta$ (iii) and IL-6 mRNA expression (iv) in the indicated treatment groups. (b) Representative confocal micrographs of serum/insulin-starved wild-type IEC-6 cells, with treatment groups as indicated, stained for pGSK3 $\beta^{\text {S9 }}$ (green) and 4',6-diamidino-2-phenylindole (DAPI; blue) (i-v). Quantification of pGSK3 ${ }^{\text {S9 }}$ staining (vi, mean pixel intensity, ImageJ Software, NIH). (c) Representative confocal micrographs of GSK3 $\beta-k d$ IEC-6 cells stained for the p65 subunit of NF-kB (green) with indicated treatment groups (i-iii). Real-time PCR (RT-PCR) showing GSK3 $\beta$ (upper) and loading control RPLO (lower) in wild-type IEC-6 cells (left lanes), GSK3 $\beta$ knockdown (GSK3 $\beta$-kd) IEC-6 cells (right lanes) (iv). Quantification of the extent of NF- $\kappa B$ translocation (v) or IL-6 mRNA expression (vi) under the indicated conditions in GSK3 $\beta$-kd IEC-6 cells. ${ }^{*} P<0.05$ vs. control (Ctrl, white bar), ${ }^{\star *} P<0.05$ vs. LPS; ${ }^{\#} P<0.05$ vs. LPS, ${ }^{\# \#} P<0.05$ vs. LPS + BM, ${ }^{\# \# \#} P<0.05$ vs. LPS + EGFimmunodepleted breast milk (IBM). Results are representative of three separate experiments with over 50 high-power fields per group. Scale bar $=10 \mu \mathrm{m}$. Data are mean \pm s.e.m.

\section{DISCUSSION}

In this study, we demonstrate a mechanism by which breast milk inhibits the LPS-mediated activation of TLR4 and the ensuing inflammatory cascade leading to protection against
NEC. We show that breast milk inhibits TLR4-mediated inflammatory signaling in vitro and that these effects are dependent on EGFR signaling via the downstream target GSK3 $\beta$. Furthermore, we discovered that breast milk attenuates 
a
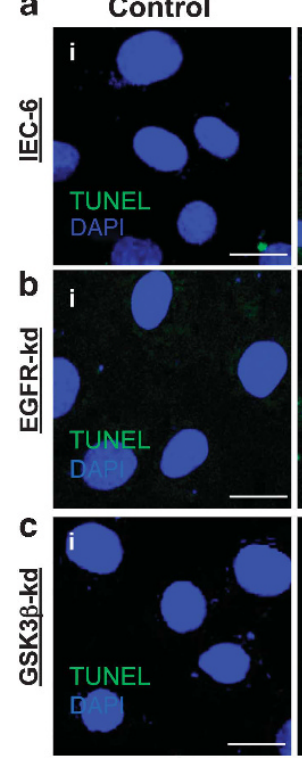

d i
LPS
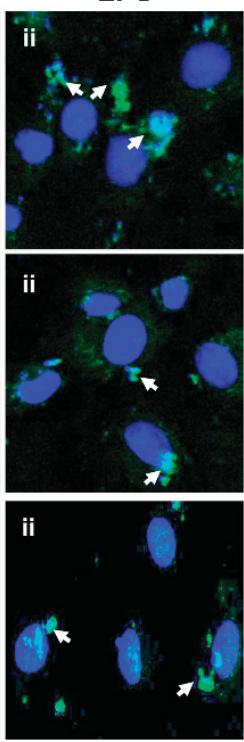

LPS+BM
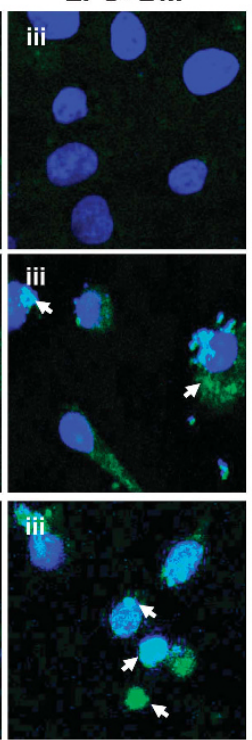

LPS+IBM
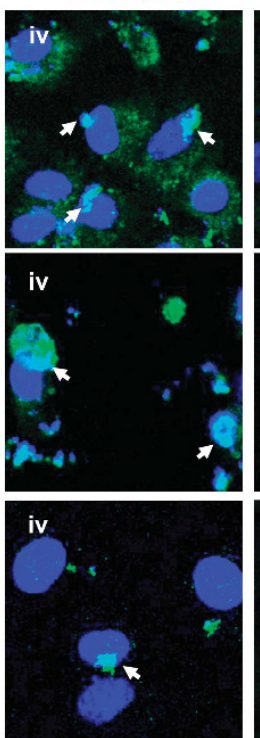

LPS+IBM+EGF
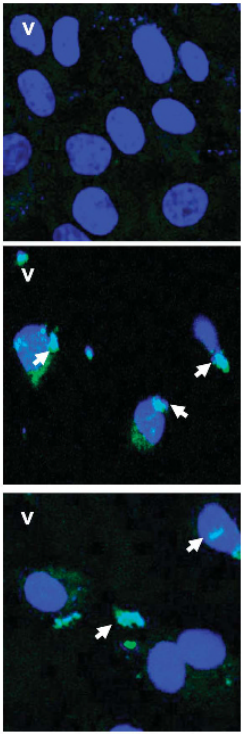
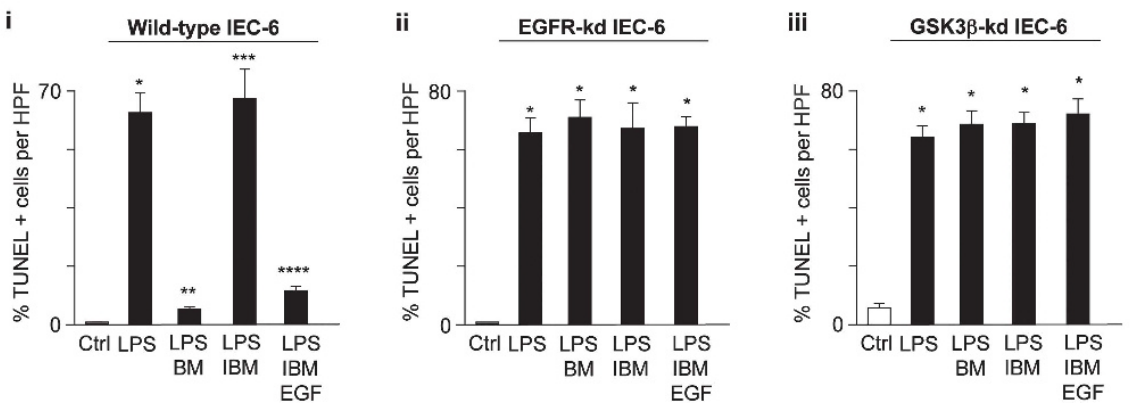

Figure 6 Breast milk (BM) inhibits Toll-like receptor 4 (TLR4)-mediated apoptosis via epidermal growth factor receptor (EGFR) and glycogen synthase kinase-3 $\beta$ (GSK3 $\beta$ ). (a-c) Representative confocal micrographs of either wild-type IEC-6 cells (ai-v) or EGFR-kd IEC-6 (bi-v) or GSK3 $\beta$-kd IEC-6 cells $(\mathbf{c i}-\mathrm{v})$ that were serum starved $6 \mathrm{~h}$, treated as indicated, then stained for terminal deoxynucleotidyl transferase dUTP nick end labeling (TUNEL; green, arrows) and 4',6-diamidino-2-phenylindole (DAPI; blue). (d) Quantification of the TUNEL-positive cells per high-power field (HPF) in wild-type IEC-6 cells (i) or EGFR-kd IEC-6 (ii) or GSK3 $\beta$-kd IEC-6 cells (iii). ${ }^{\star} P<0.05$ vs. control (Ctrl, white bar), ${ }^{* \star} P<0.05$ vs. lipopolysaccharide (LPS), ${ }^{\star \star \star} P<0.05$ vs. LPS + BM, and ${ }^{* \star * \star} P<0.05$ vs. LPS + immunodepleted breast milk (IBM). Results are representative of three separate experiments with over 50 HPFs per group. Scale bar $=10 \mu \mathrm{m}$. Data are mean \pm s.e.m. Arrows delineate apoptotic cells.

experimental NEC via inhibition of TLR4 signaling through EGF, as treatment with EGF-deficient breast milk abrogated the protective effects that were restored with exogenous EGF added back to the formula, thus confirming the role of EGF on the prevention of NEC. Further support of the mechanism that EGF and its receptor EGFR are critical components mediating the protection against NEC is demonstrated by the current observation that mice in which EGFR had been deleted specifically from the intestinal epithelium were not protected from experimental NEC when breast milk was added to their formula. Taken together, these findings present a new paradigm to explain the protective effects of breast milk on TLR4 signaling via effects on a growth factor receptor.

Inhibition of TLR4 activation within the intestinal epithelium has been shown to enhance enterocyte proliferation $^{7,19}$ and to inhibit enterocyte apoptosis in the small intestine of the premature host. ${ }^{21,27}$ We have previously shown an important link between TLR 4 and the GSK3 $\beta / \beta$-catenin signaling pathway in NEC pathogenesis. ${ }^{7}$ TLR4 activation was found to increase GSK3 $\beta$ expression, resulting in enhanced phosphorylation of $\beta$-catenin and its targeted degradation, culminating in reduced enterocyte proliferation. ${ }^{7}$ We now demonstrate that in the pathogenesis of NEC, breast milk and EGF have protective roles on the intestinal mucosa via inhibition of enterocyte apoptosis and enhanced intestinal mucosal healing, providing a potential link to our prior findings. Specifically, the GSK3 $\beta / \beta$-catenin signaling pathway plays a critical role in determining the extent of enterocyte proliferation that occurs in response to EGFR activation. ${ }^{32}$ Furthermore, growth factor receptor/ligand interaction initiates a signaling cascade that affects GSK3 $\beta$ that modulates TLR4-mediated NF- $\kappa \mathrm{B}$ activity to transition between the generation of pro- and anti-inflammatory signals. ${ }^{43-45}$ Inactivation of GSK3 $\beta$ by phosphorylation at serine 9 negatively affects NF- $\kappa \mathrm{B}$ activation, ${ }^{43-45}$ thus decreasing NF- $\kappa \mathrm{B}$-dependent pro-inflammatory cytokine production. ${ }^{44}$ Moreover, 
a
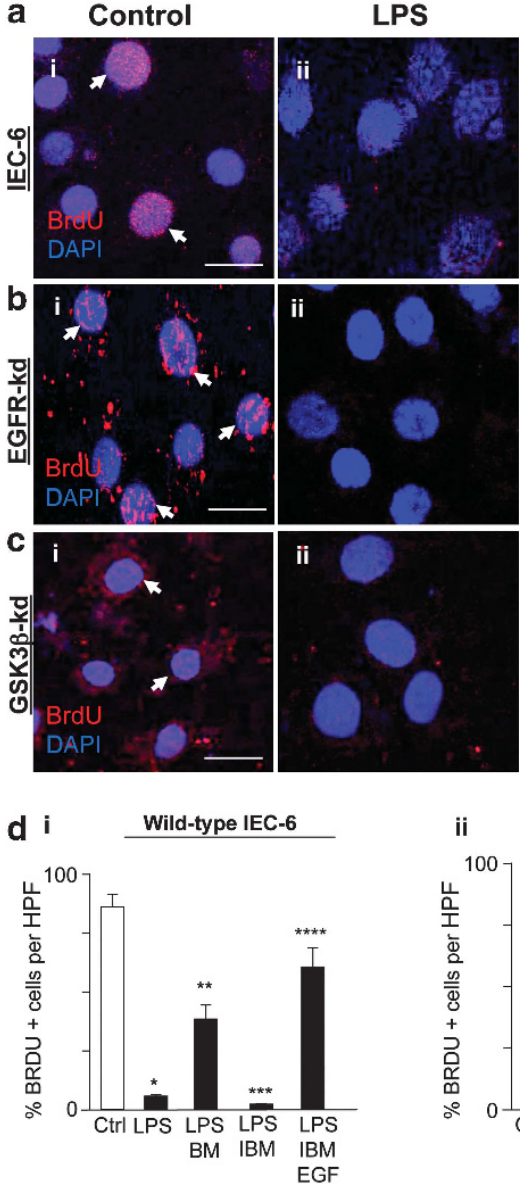

LPS
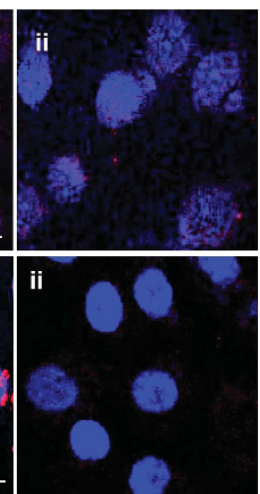

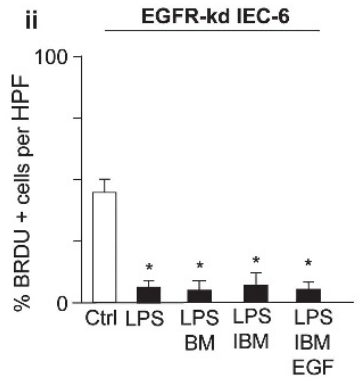

LPS+BM
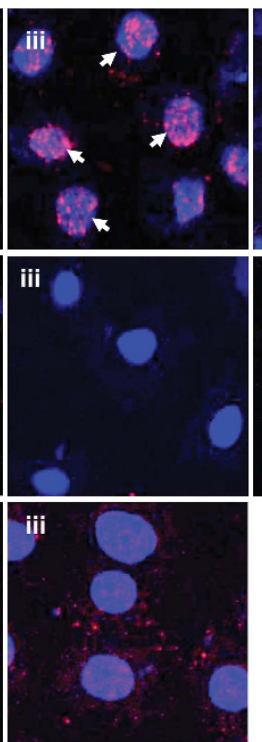
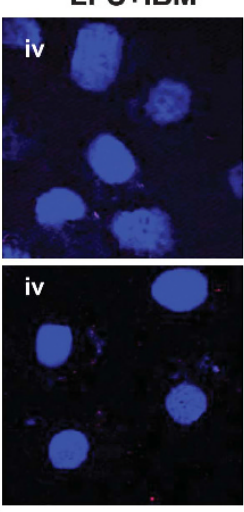

LPS+IBM+EGF
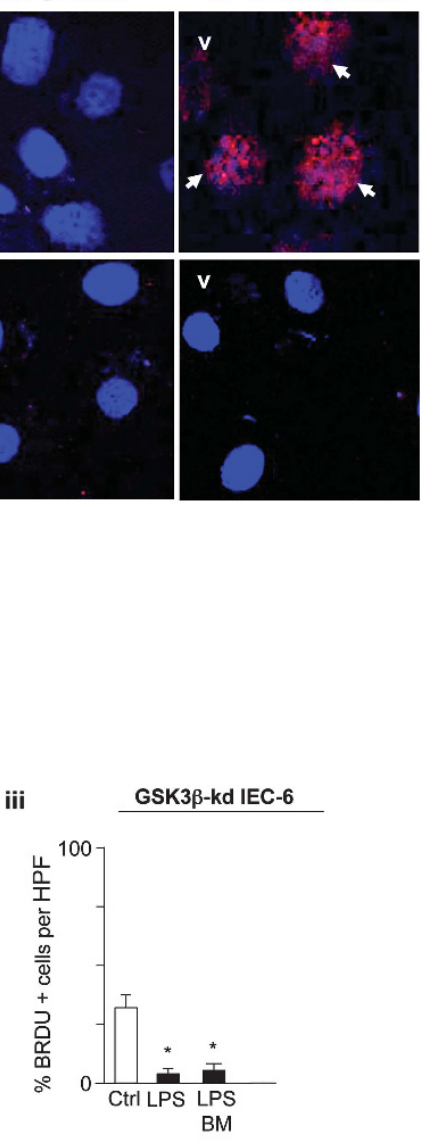

Figure 7 Breast milk (BM) restores enterocyte proliferation via epidermal growth factor receptor (EGFR) and glycogen synthase kinase-3 $\beta$ (GSK3 3 ). (a-c) Representative confocal micrographs of either wild-type IEC-6 cells (ai-v) or EGFR-kd IEC-6 (bi-v) or GSK3 $\beta$-kd IEC-6 cells (ci-iii), treated as indicated, then stained for the proliferation marker bromodeoxyuridine (BrdU, red, arrows) and 4',6-diamidino-2-phenylindole (DAPI; blue). (d) Quantification of the BrdU-positive cells per high-power field (HPF) in wild-type IEC-6 cells (i) or EGFR-kd IEC-6 (ii) or GSK3 $\beta$-kd IEC-6 cells (iii). ${ }^{\star} P<0.05$ vs. control (Ctrl, white bar), ${ }^{\star \star} P<0.05$ vs. lipopolysaccharide (LPS), ${ }^{\star \star \star} P<0.05$ vs. LPS + BM, and ${ }^{\star \star \star \star} P<0.05$ vs. LPS + immunodepleted breast milk (IBM). Results are representative of three separate experiments with over 50 HPFs per group. Scale bar $=10 \mu \mathrm{m}$. Data are mean \pm s.e.m. Arrows delineate proliferative cells.

inactivation of GSK3 $\beta$ also leads to the stabilization of $\beta$-catenin, a critical factor responsible for growth and proliferation. $^{32,46}$ Moreover, although peroxisome proliferator-activated receptor- $\gamma$ has been linked to EGFR receptor signaling, we have previously demonstrated that deletion of peroxisome proliferator-activated receptor- $\gamma$ in intestinal epithelial cells had no effect on EGFR expression, raising the possibility that other effector mechanisms are involved. ${ }^{19}$ By extension, given that breast milk is rich in EGF, it is possible that the EGF in breast milk could play similar roles via GSK3 $\beta$ / $\beta$-catenin signaling and inactivation of GSK $3 \beta,{ }^{19}$ although it appears now that effects of EGFR activation on TLR4 expression predominate.

Our data are consistent with previous studies that have demonstrated an important role for EGF in reducing the incidence of NEC development. ${ }^{16,17}$ In addition, a recent study has shown that a low concentration of epidermal growth factor in the cord blood of extremely premature infants is associated with the development of NEC. ${ }^{47}$ We now extend these findings by providing a novel mechanistic link associated with breast milk-mediated protection in NEC via EGF. Other compounds are known to be present in breast milk and may also be important in the regulation of NEC pathogenesis. These include human milk oligosaccharides that are nondigestible carbohydrates thought to influence the gastrointestinal microbiome $^{48}$ and possibly protect against NEC. ${ }^{49}$ Besner and colleagues $^{50,51}$ have evaluated the effects of heparin-binding epidermal growth factor-like growth factor on experimental NEC and have shown that it is protective by accelerating enterocyte migration and proliferation, along with increasing the microvascular blood flow of the intestine. These studies demonstrate the clinical importance of identifying the components of breast milk that are protective against NEC, so as to provide potential therapeutic targets for this devastating disease. We readily acknowledge that although we provide here a mechanism by which breast milk attenuates experimental NEC, there may be other signaling pathways involved in the protection against NEC, such as autophagy, the mammalian 
a
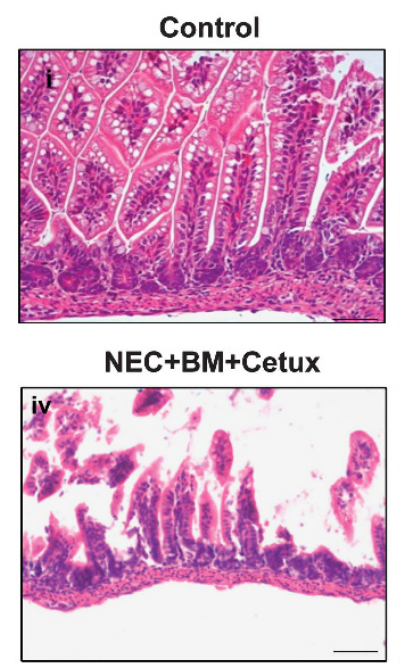

b

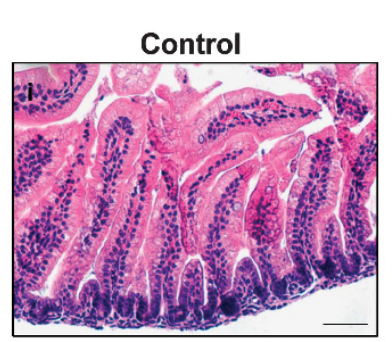

Wild-type

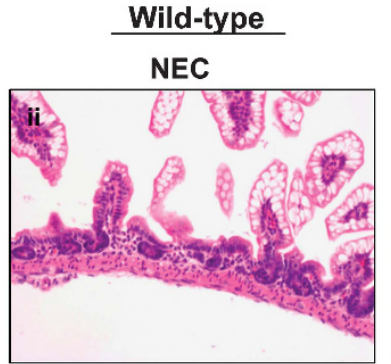

NEC+IBM

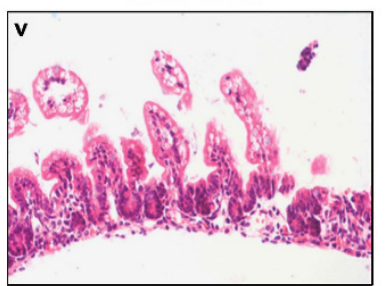

EGFR $^{\triangle I E C}$

NEC

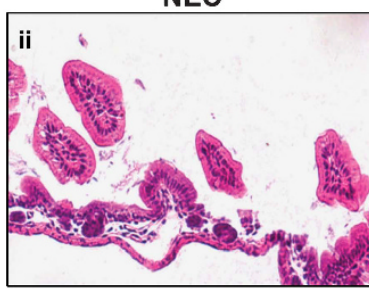

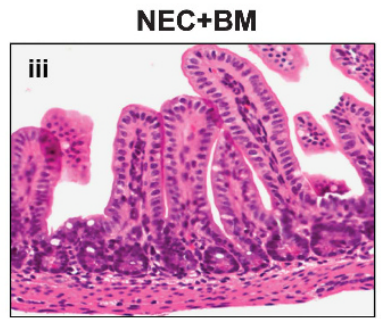

NEC+IBM+EGF
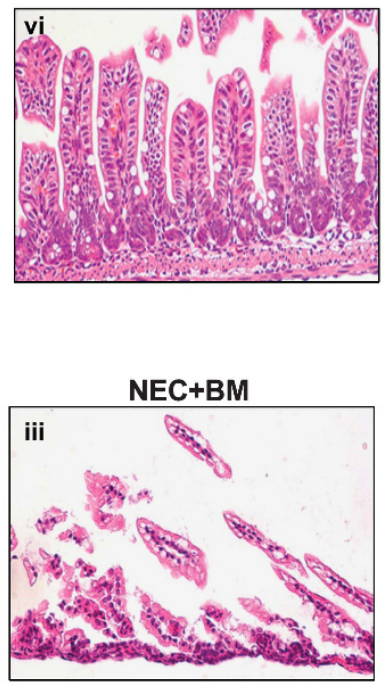

$E^{E F R}{ }^{\triangle I E C}$
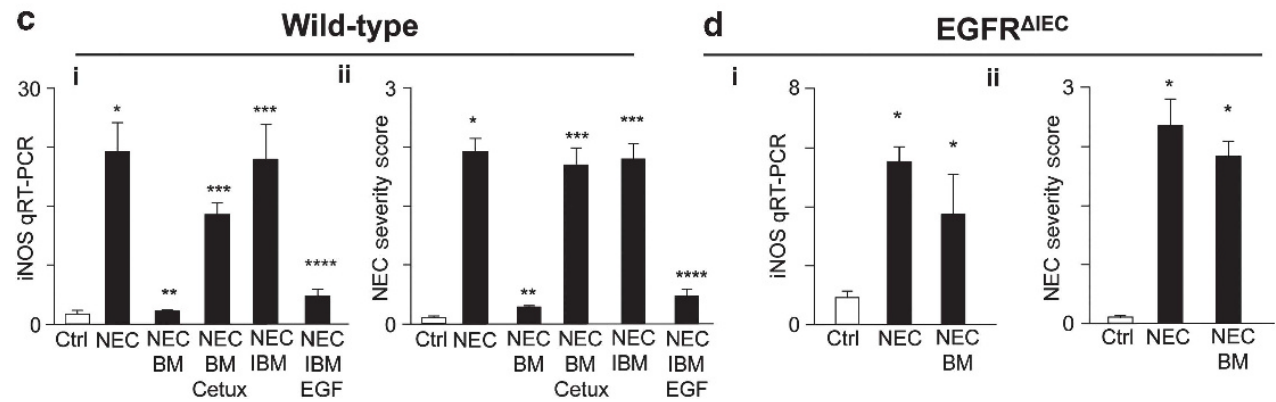

Figure 8 Breast milk (BM) attenuates necrotizing enterocolitis (NEC) severity via epidermal growth factor receptor (EGFR) activation. (a, b) Representative hematoxylin and eosin (H\&E) micrographs of the terminal ileum of either wild-type mice (ai-vi) or EGFR ${ }^{\Delta \mathrm{IEC}}$ mice (bi-iii) for the indicated experimental NEC treatment groups. Cetux, cetuximab. (c, d) Inducible nitric oxide synthase (iNOS) mRNA expression for indicated groups in wild-type mice (ci) or EGFR ${ }^{\Delta I E C}$ mice (di) and NEC severity score assigned by a pathologist blinded to the treatment groups in wild-type mice (cii) or EGFR ${ }^{\Delta I E C}$ mice (dii). ${ }^{\star} P<0.05$ vs. breast-fed control animals (Ctrl, white bar), ${ }^{\star \star} P<0.05$ vs. NEC, ${ }^{* * *} P<0.05$ vs. NEC + BM, and ${ }^{* * *} P<0.05$ vs. NEC + EGFimmunodepleted breast milk (IBM). Data are mean \pm s.e.m. Results are representative of at least three separate experiments with at least 10 mice per group.

target of rapamycin pathway, or inhibition of endoplasmic reticulum stress. Further studies will be required to explore the potential roles - if any-of these varying signaling pathways in mediating the observed protection of breast milk in infants with NEC.

In summary, we have now described a mechanistic pathway to explain how breast milk mediates protection against the development of NEC in mice. Further studies into how breast milk offers such profound protection against the development of this disease-with a focus on the anti-inflammatory properties of breast milk-may open doors toward novel approaches for the rational design of new therapies of this devastating condition.

\section{METHODS}

Reagents. Antibodies were obtained from the following sources: p65 subunit of NF- $\kappa B$ and phospho and total GSK3 $\beta$ (Cell Signaling, Danvers, MA); proliferating cell nuclear antigen (Santa Cruz, Dallas, TX); 3-nitrotyrosine (Abcam, Cambridge, MA), actin (Genscript, Piscataway, NJ); bromodeoxyuridine (BrdU, Novus Biosciences, Littleton, CO); and 4',6-diamidino-2-phenylindole (Invitrogen, Grand Island, NY). TUNEL (Roche Applied Science, Indianapolis, IN) was performed according to the manufacturer's instructions as in 

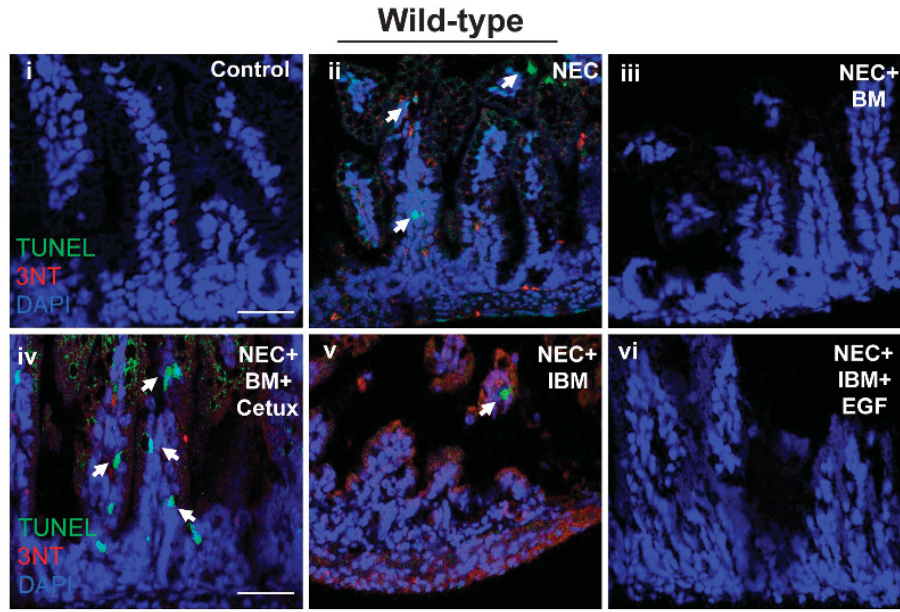

b
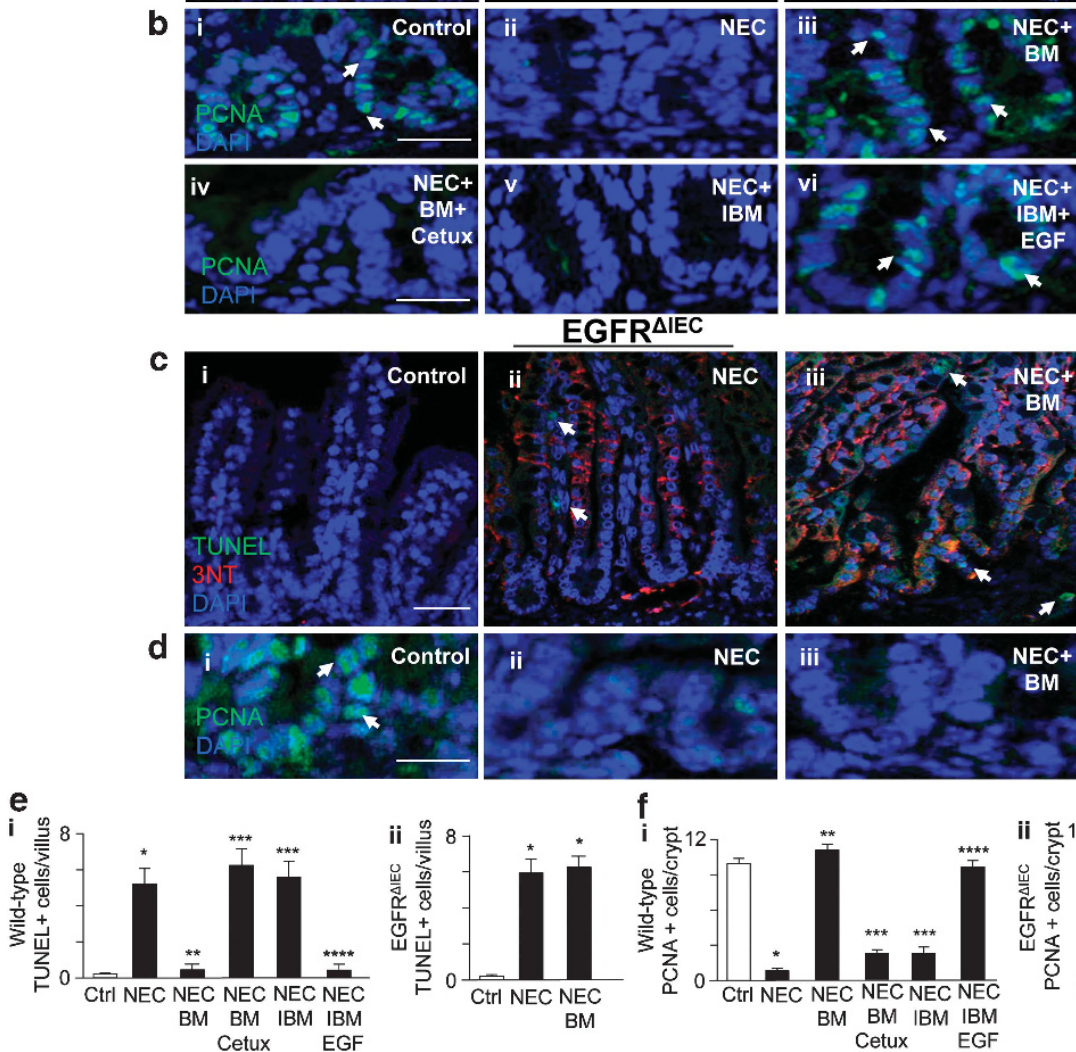

EGFR ${ }^{\Delta I E C}$

NEC
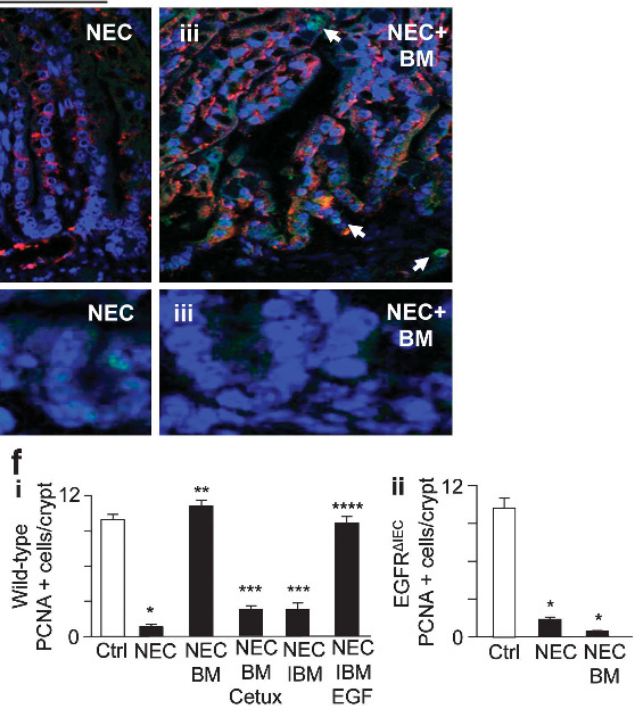

Figure 9 Breast milk (BM) inhibits necrotizing enterocolitis (NEC)-mediated apoptosis and enhances crypt proliferation via epidermal growth factor/ epidermal growth factor receptor (EGF/EGFR). (a) Representative confocal micrographs of the terminal ileum of wild-type mice stained for terminal deoxynucleotidyl transferase dUTP nick end labeling (TUNEL; green, arrows), 3-nitrotyrosine (3NT, red), and 4',6-diamidino-2-phenylindole (DAPI; blue) from the indicated experimental NEC treatment groups (i-vi). (b) Representative confocal micrographs of the small intestinal crypts of wild-type mice stained for proliferating cell nuclear antigen (PCNA; green, arrows) and DAPI (blue) from the indicated experimental NEC treatment groups (i-vi). (c) Representative confocal micrographs of the terminal ileum of EGFR ${ }^{\Lambda I E C}$ mice stained for TUNEL (green, arrows), 3NT (red), and DAPI (blue) from the indicated experimental NEC treatment groups (i-iii). (d): Representative confocal micrographs of the small intestinal crypts of wild-type mice stained for PCNA (green, arrows) and DAPI (blue) from the indicated experimental NEC treatment groups (i-iii). (e) Quantification of the TUNEL-positive cells per villus in wild-type mice (i) or EGFR ${ }^{\Delta I E C}$ mice (ii). (f) Quantification of the PCNA-positive cells per crypt in wild-type mice (i) or EGFR ${ }^{\Delta I E C}$ mice (ii). ${ }^{*} P<0.05$ vs. breast-fed control animals (Ctrl, white bar), ${ }^{\star \star} P<0.05$ vs. NEC, ${ }^{* *} P<0.05$ vs. NEC $+\mathrm{BM}$, and ${ }^{\star \star * \star} P<0.05$ vs. NEC + EGF-immunodepleted breast milk (IBM). Data are mean \pm s.e.m. Results are representative of at least three separate experiments with at least 10 mice per group.

Afrazi et al. $^{21}$ Appropriate secondary antibodies for immunohistochemistry and SDS-PAGE were obtained from Molecular Probes (Grand Island, NY) and Jackson ImmunoResearch Laboratories (West Grove, PA). Cetuximab (dose: $100 \mu \mathrm{g}$ per day intraperitoneally for 3 days before experiments) was a generous gift of Dr. Jennifer Grandis (University of Pittsburgh, Pittsburgh, PA). Human recombinant EGF was from EMD Millipore (Billerica, MA) $\left(400 \mathrm{ng} \mathrm{ml}^{-1}\right.$ media in vitro, $0.5 \mathrm{ng}$ per $\mu \mathrm{l}$ of NEC formula in vivo). Lithium chloride was from J.T. Baker (Avantor, Center Valley, PA) $(100 \mu \mathrm{M})$. LPS (Escherichia coli 0111:B4 purified by gel-filtration chromatography, $>99 \%$ pure) was obtained from Sigma-Aldrich (St. Louis, MO) and concentrations used were those that we have demonstrated to be present in mice and 
Table 1 List of primers

\begin{tabular}{|c|c|c|c|c|}
\hline Gene & Species & Forward sequence $\left(5^{\prime}-3^{\prime}\right)$ & Reverse sequence $\left(5^{\prime}-3^{\prime}\right)$ & Amplicon Size (bp) \\
\hline IL-6 & Mouse/rat & GGCTAAGGACCAAGACCATCCAA & TCTGACCACAGTGAGGAATGTCCA & 138 \\
\hline$I L-1 \beta$ & Mouse/rat & AGTGTGGATCCCAAGCAATACCCA & TGTCCTGACCACTGTTGTITCCCA & 175 \\
\hline iNOS & Mouse/rat & CTGCTGGTGGTGACAAGCACATT & ATGTCATGAGCAAAGGCGCAGAAC & 167 \\
\hline GAPDH & Mouse/rat & TGAAGCAGGCATCTGAGGG & CGAAGGTGGAAGAGTGGGAG & 102 \\
\hline$E G F R-k d$ & Mouse/rat & ATGGTGTCACTGTGTGGGAACTGA & ACTITGGGCGGCTATCAGCATCTA & 183 \\
\hline EGFR K/O gt & Mouse & CTCAGCCAGATGATGTTGAC & CCTCGTCTGTGGAAGAACTA & 129 \\
\hline GSK3 $\beta-k d$ & Rat & GACACACCTGCCCTCTTCAA & AGAAGCGGCGTTATTGGTCT & 177 \\
\hline
\end{tabular}

humans with NEC ( $50 \mu \mathrm{g} \mathrm{ml}^{-1}$ for cells, $5 \mathrm{mg} \mathrm{kg}^{-1}$ for all mice ${ }^{3}$ with the exception of the TLR4 ${ }^{\triangle \mathrm{IEC}-\mathrm{OVER}}$ mice who are sensitive to LPS and required a decreased dosage $\left.\left(2.5 \mathrm{mg} \mathrm{kg}^{-1}\right)\right)$.

Cell culture and lentiviral knockdown. The small intestinal cell line, IEC-6, was obtained from the American Type Culture Collection (Manassas, VA), maintained as in Quaroni et al., ${ }^{52}$ and rendered deficient in EGFR or GSK3 $\beta$ using lentiviral particles containing EGFR or GSK3 $\beta$ short hairpin RNA (Open Biosystems, Lafayette, CO) from the four-plasmid lentiviral packaging system (Invitrogen, Grand Island, NY) with permissive HEK 293 cells. In parallel, IEC-6 cells were treated with lentiviruses expressing scrambled short hairpin RNA. Stable integration of lentivirus was obtained by selection of cells in medium containing puromycin $\left(5 \mu \mathrm{g} \mathrm{ml}^{-1}\right)$, and the extent of knockdown of EGFR or GSK3 $\beta$ was verified by real-time PCR or SDSPAGE as in Good et al. ${ }^{19}$ Serum and/or insulin starvation of the cells for $6 \mathrm{~h}$ was performed where indicated in the figure legends.

Quantitative real-time PCR. Quantitative real-time PCR with the BioRad CFX96 Real-Time System (Bio-Rad, Hercules, CA) was performed as in Afrazi et al. ${ }^{21}$ with the following primers using either RPLO or GAPDH as a housekeeping gene as shown in Table 1.

Statement of ethics. The animal experiments described in these studies were approved by the University of Pittsburgh Animal Care and Use Committee (Protocol Number: 12040382) and were performed in strict accordance with the recommendations in the Guide for the Care and Use of Laboratory Animals of the National Institutes of Health.

Collection of mouse breast milk. We obtained breast milk from isoflurane-anesthesized lactating mice on postpartum days 8-12 as in Yazji et al. ${ }^{8}$ that had been injected with oxytocin $(0.15 \mathrm{IU}$ subcutaneous per $\mathrm{kg}$ of body weight for $3 \mathrm{~min}$ before milk being collected via an electronic breast pump (Lansinoh Laboratories, Alexandria, VA). We typically obtain $\sim 1 \mathrm{ml}$ per mouse that was immediately aliquoted and stored at $-80^{\circ} \mathrm{C}$ and frozen just once before use.

Induction of experimental murine endotoxemia and whole-animal NF- $\kappa \mathbf{B}$ signaling. Wild-type mice were obtained from either Jackson Laboratory (Bar Harbor, ME) or Charles River Laboratories (Wilmington, MA). After being placed nil per os for $6 \mathrm{~h}$, endotoxemia was induced in 10-day-old mice with intraperitoneal injection of LPS (5 $\left.\mathrm{mg} \mathrm{kg}^{-1}, 6-16 \mathrm{~h}\right)$. In parallel, mice were administered saline as vehicle control. Where indicated, mice were gavaged expressed murine breast milk or saline as a control ( $50 \mu \mathrm{l}$ per g of mouse body weight) as a pretreatment $1 \mathrm{~h}$ before LPS injection. Mice lacking EGFR specifically in the intestinal epithelium were generated by breeding Egfr loxP/loxP (obtained from David W. Threadgill, University of North Carolina, Chapel Hill, NC) with transgenic mice expressing Villin-cre (termed
$\mathrm{EGFR}^{\mathrm{AIEC}}$ mice). Mice were generated to selectively express TLR4 within in the intestinal epithelium (TLR4 $4^{\triangle \mathrm{IEC}-\mathrm{OVER}}$ ) as in Afrazi et al. ${ }^{21}$ Experiments with the NF- $\kappa \mathrm{B}$-luciferase reporter mice in which NF- $\kappa \mathrm{B}$ is upstream of the luciferase gene (strain NF- $\kappa \mathrm{B}-\mathrm{RE}-\mathrm{luc}$, Taconic Farms, Hudson, NY) were performed as in Neal et al. ${ }^{9}$ and imaged using the IVIS Lumina 3D Optimal in vivo imaging system (Waltham, MA) $10 \mathrm{~min}$ after an intraperitoneal injection of luciferin $\left(150 \mu \mathrm{g} \mathrm{kg}^{-1}\right.$, Caliper Life Sciences, Hopkinton, MA). Samples of the terminal ileum were obtained $1 \mathrm{~cm}$ proximal to the ileocecal valve and prepared as described in Leaphart et al. ${ }^{3}$

Induction of experimental murine necrotizing enterocolitis. Experimental NEC was induced in 5-10-day old mice as described previously. ${ }^{19,21,42}$ Briefly, NEC formula (Similac Advance infant formula (Abbott Nutrition, Columbus, OH): Esbilac (PetAg, Hampshire, IL) canine milk replacer, 2:1) was supplemented with enteric bacteria made from a stock created from a specimen obtained from an infant with surgical NEC ( $12.5 \mu$ loriginal stool slurry in $1 \mathrm{ml}$ formula) and was orally gavage fed five times per day ( $50 \mu \mathrm{l}$ per g of mouse body weight) as in Good et al. ${ }^{42}$ The mice also received hypoxia for 10 min twice daily ( $5 \%$ $\mathrm{O}_{2}, 95 \% \mathrm{~N}_{2}$ ) in a hypoxic chamber (Billups-Rothenberg, Inc., Del Mar, CA) for a total of 4 days. We have shown that this experimental NEC protocol induces intestinal inflammation and the release of proinflammatory cytokines that closely resembles human NEC., ${ }^{3,421,42}$ Control (i.e., non-NEC) animals remained with their mothers and were breast fed on demand. Where indicated, formula-fed mice were enterally administered expressed murine breast milk that was obtained as in Yazji et $a .^{8}$ or EGF-deficient breast milk once per day with or without recombinant EGF ( $0.5 \mathrm{ng} \mathrm{hl}^{-1}$ of formula) added back to that feeding only. The expression of mucosal cytokines was assessed by quantitative real-time PCR as in Good et al. ${ }^{42}$ Immediately after killing, the terminal ileum was harvested $1 \mathrm{~cm}$ proximal to the ileocecal valve and fixed in $4 \%$ paraformaldehyde. The severity of disease was determined on histological sections of the terminal ileum by a pediatric pathologist who was blinded to the study conditions according to our previously published scoring system from 0 (normal) to 3 (severe). ${ }^{3}$

Immunohistochemistry, immunofluorescence, and SDS-PAGE. Immunohistochemistry, immunofluorescence, and confocal microscopy was performed as in Good et al. ${ }^{19}$ except for in vitro BrdU proliferation assay that is described below. Images were assembled using Adobe Photoshop CS6 software (Adobe Systems, San Jose, CA). SDS-PAGE was performed as in Good et al. ${ }^{19}$ and the densitometry quantified with ImageJ Software (NIH, Bethesda, MD).

In Vitro BrdU proliferation assay. To assess proliferative S-phase cells, wild-type IEC-6, EGFR-kd IEC-6, or GSK3 $\beta$-kd IEC-6 cells were plated on cover slips in 12-well cell culture dishes overnight, treatment 
groups as indicated in Results along with BrdU labeling reagent $\left(10 \mu \mathrm{l} \mathrm{ml}{ }^{-1}\right.$ media, Invitrogen) for $6 \mathrm{~h}$. To detect BrdU incorporation, cells were fixed with $4 \%$ paraformaldehyde on ice for $10 \mathrm{~min}$, processed for antigen retrieval with $2 \mathrm{~m}$ hydrochloric acid for $30 \mathrm{~min}$, blocked with $5 \%$ donkey serum in $0.5 \%$ bovine serum albumin, incubated overnight at $4{ }^{\circ} \mathrm{C}$ with rat-anti-BrdU antibodies (Novus Biosciences), and detected with cy3-labeled secondary anti-rat antibodies using a confocal microscope (Zeiss, Dublin, CA).

Assessment of NF- $\kappa$ B activation in enterocytes. Cultured wild-type IEC- 6 cells or cells in which knockdown of EGFR or GSK3 $\beta$ IEC- 6 cells were treated with LPS $\left(50 \mu \mathrm{g} \mathrm{ml}^{-1}, 1 \mathrm{~h}\right)$ after pretreatments of murine breast milk ( $25 \mu \mathrm{l}$ per well, $1 \mathrm{~h}$ ) or EGF $\left(400 \mathrm{ng} \mathrm{ml}^{-1}, 1 \mathrm{~h}\right)$. The extent of NF- $\kappa \mathrm{B}$ translocation was determined as in Good et al. ${ }^{19}$ and the average integrated pixel intensity that pertained to the corresponding $\mathrm{NF}-\kappa \mathrm{B}$ staining within the cytoplasmic and nuclear regions was determined for $>200$ cells per treatment group in at least three experiments per group using MetaMorph software version 6.1 (Molecular Devices, Downingtown, PA).

Where indicated, the extent of apoptosis was quantified in vitro and in vivo as we have done previously ${ }^{21}$ using the TUNEL assay and enumerating the number of TUNEL-positive cells as a percentage of the total number of cells present. At least 100 fields were assessed for each experimental group where indicated.

Immunodepletion of EGF from breast milk. Immunodepletion of EGF from breast milk was performed as in Good et al. ${ }^{19}$ Briefly, $500 \mu \mathrm{l}$ aliquots of freshly harvested murine breast milk were filtered via Amicon Ultra 10K Centrifugal Filters (Millipore, Billerica, MA) at 13,000 r.p.m. for $15 \mathrm{~min}$. Filtered aliquots were then incubated at $4{ }^{\circ} \mathrm{C}$ for $30 \mathrm{~min}$ with control goat IgG antibody $(10 \mu \mathrm{l})$ and protein A/Gagarose $(20 \mu$ l, Santa Cruz Biotechnology), and centrifuged at 3,000 r.p.m. for $30 \mathrm{~s}$. The supernatant was incubated for $1 \mathrm{~h}$ at $4{ }^{\circ} \mathrm{C}$ with $2 \mu \mathrm{g}$ of anti-EGF antibody (Santa Cruz Biotechnology) followed by overnight incubation on a rotator with an additional $20 \mu \mathrm{l}$ of protein A/ G-agarose. Supernatants were collected after centrifugation at 3,000 r.p.m. for $30 \mathrm{~s}$ and an EGF ELISA (Abcam) was performed as per the manufacturer's instructions using a 1:500 dilution (wild-type murine breast milk 22,907 $\pm 976 \mathrm{pg} \mathrm{ml}^{-1}$; EGF-depleted murine breast milk $468.5 \pm 87.1 \mathrm{pg} \mathrm{ml}^{-1}$ ).

Statistical analysis. Statistical analysis was performed using PRISM 6 (GraphPad, La Jolla, CA) software and analysis of variance, $\chi^{2}$ test, or two-tailed Student's $t$-test were used for comparisons where indicated. Data are mean \pm or s.e.m. as indicated, and comparisons are by twotailed Student's $t$-test or analysis of variance with statistical significance at a $P$-value of $<0.05$. All experiments were repeated at least in triplicate, with more than 50-200 cells/high-power field for in vitro experiments. For in vivo experiments, at least 3 mice per group for endotoxemia and at least 10 pups per group for experimental NEC were assessed.

\section{ACKNOWLEDGMENTS}

M.G. is supported by K08DK101608 from the National Institutes of Health and the Children's Hospital of Pittsburgh of the UPMC Health System. D.J.H. is supported by R01GM078238 and R01DK083752 from the National Institutes of Health.

\section{DISCLOSURE}

The authors declared no conflict of interest.

(c) 2015 Society for Mucosal Immunology

\section{REFERENCES}

1. Neu, J. \& Walker, W.A. Necrotizing enterocolitis. N. Engl. J. Med. 364 255-264 (2011).
2. Henry, M.C. \& Moss, R.L. Necrotizing enterocolitis. Annu. Rev. Med. 60, 111-124 (2009).

3. Leaphart, C.L. et al. A critical role for TLR4 in the pathogenesis of necrotizing enterocolitis by modulating intestinal injury and repair. J. Immunol. 179, 4808-4820 (2007).

4. Sodhi, C.P. et al. Intestinal epithelial Toll-like receptor 4 regulates goblet cell development and is required for necrotizing enterocolitis in mice. Gastroenterology 143, 708-718 e701-705 (2012).

5. Jilling, T. et al. The roles of bacteria and TLR4 in rat and murine models of necrotizing enterocolitis. J. Immunol. 177, 3273-3282 (2006).

6. Richardson, W.M. et al. Nucleotide-binding oligomerization domain-2 inhibits toll-like receptor-4 signaling in the intestinal epithelium. Gastroenterology 139, 904-917. 917.e1-6 (2010).

7. Sodhi, C.P. et al. Toll-like receptor-4 inhibits enterocyte proliferation via impaired beta-catenin signaling in necrotizing enterocolitis. Gastroenterology 138, 185-196 (2010).

8. Yazji, I., Sodhi, C.P., Lee, E.K., Good, M., Egan, C.E. \& Afrazi, A. et al. Endothelial TLR4 activation impairs intestinal microcirculatory perfusion in necrotizing enterocolitis via eNOS-NO-nitrite signaling. Proceedings of the National Academy of Sciences of the United States of America 2013.

9. Neal, M.D. et al. Discovery and validation of a new class of small molecule Toll-like receptor 4 (TLR4) inhibitors. PLoS One 8, e65779 (2013).

10. Gephart, S.M., McGrath, J.M., Effken, J.A. \& Halpern, M.D. Necrotizing enterocolitis risk: state of the science. Adv. Neonatal Care 12, 77-87. quiz 88-79 (2012).

11. Lucas, A. \& Cole, T.J. Breast milk and neonatal necrotising enterocolitis. Lancet 336, 1519-1523 (1990).

12. Newburg, D.S. \& Walker, W.A. Protection of the neonate by the innate immune system of developing gut and of human milk. Pediatr. Res. 61, 2-8 (2007).

13. Meinzen-Derr, J., Poindexter, B., Wrage, L., Morrow, A.L., Stoll, B. \& Donovan, E.F. Role of human milk in extremely low birth weight infants' risk of necrotizing enterocolitis or death. J. Perinatol. 29, 57-62 (2009).

14. Pollack, P.F. et al. Effects of enterally fed epidermal growth factor on the small and large intestine of the suckling rat. Regul. Pept. 17, 121-132 (1987).

15. Dvorak, B. et al. Milk-borne epidermal growth factor modulates intestinal transforming growth factor-alpha levels in neonatal rats. Pediatr. Res. 47 194-200 (2000).

16. Dvorak, B. et al. Epidermal growth factor reduces the development of necrotizing enterocolitis in a neonatal rat model. Am. J. Physiol. Gastrointest. Liver Physiol. 282, G156-G164 (2002).

17. Clark, J.A. et al. Intestinal barrier failure during experimental necrotizing enterocolitis: protective effect of EGF treatment. Am. J. Physiol. Gastrointest. Liver Physiol. 291, G938-G949 (2006).

18. Hirai, C., Ichiba, H., Saito, M., Shintaku, H., Yamano, T. \& Kusuda, S. Trophic effect of multiple growth factors in amniotic fluid or human milk on cultured human fetal small intestinal cells. J. Pediatr. Gastroenterol. Nutrit. 34, 524-528 (2002).

19. Good, M. et al. Amniotic fluid inhibits Toll-like receptor 4 signaling in the fetal and neonatal intestinal epithelium. Proc. Natl. Acad. Sci. USA 109, 11330-11335 (2012).

20. Neal, M.D. et al. Toll-like receptor 4 is expressed on intestinal stem cells and regulates their proliferation and apoptosis via the p53 up-regulated modulator of apoptosis. J. Biol. Chem. 287, 37296-37308 (2012).

21. Afrazi, A. et al. Toll-like receptor 4-mediated endoplasmic reticulum stress in intestinal crypts induces necrotizing enterocolitis. J. Biol. Chem. 289, 9584-9599 (2014)

22. Chan, K.L., Wong, K.F. \& Luk, J.M. Role of LPS/CD14/TLR4-mediated inflammation in necrotizing enterocolitis: pathogenesis and therapeutic implications. World J. Gastroenterol. 15, 4745-4752 (2009).

23. Liu, Y. et al. Changes in intestinal Toll-like receptors and cytokines precede histological injury in a rat model of necrotizing enterocolitis. Am. J. Physiol. Gastrointest. Liver Physiol. 297, G442-G450 (2009).

24. Neal, M.D. et al. Enterocyte TLR4 mediates phagocytosis and translocation of bacteria across the intestinal barrier. J. Immunol. 176, 3070-3079 (2006).

25. Ruemmele, F.M. et al. Lipopolysaccharide modulation of normal enterocyte turnover by toll-like receptors is mediated by endogenously produced tumour necrosis factor alpha. Gut 51, 842-848 (2002). 
26. Summers, S.T. \& Bass, B.L. Protein kinase C inhibits epidermal growth factor receptor phosphorylation in enterocytes. J. Surg. Res. 69, 208-211 (1997).

27. Neal, M.D. et al. A critical role for TLR4 induction of autophagy in the regulation of enterocyte migration and the pathogenesis of necrotizing enterocolitis. J. Immunol. 190, 3541-3551 (2013).

28. Jilling, T., Lu, J., Jackson, M. \& Caplan, M.S. Intestinal epithelial apoptosis initiates gross bowel necrosis in an experimental rat model of neonatal necrotizing enterocolitis. Pediatr. Res. 55, 622-629 (2004).

29. Ford, H., Watkins, S., Reblock, K. \& Rowe, M. The role of inflammatory cytokines and nitric oxide in the pathogenesis of necrotizing enterocolitis. J. Pediatr. Surg. 32, 275-282 (1997).

30. Cetin, S. et al. Nitric oxide inhibits enterocyte migration through activation of RhoA-GTPase in a SHP-2-dependent manner. Am. J. Physiol. Gastrointest. Liver Physiol. 292, G1347-G1358 (2007).

31. Riad, A., Bien, S., Gratz, M., Escher, F., Westermann, D. \& Heimesaat, M.M. Toll-like receptor-4 deficiency attenuates doxorubicin-induced cardiomyopathy in mice. Eur. J. Heart Fail. 10, 233-243 (2008).

32. Zheng, H., Saito, H., Masuda, S., Yang, X. \& Takano, Y. Phosphorylated GSK3beta-ser9 and EGFR are good prognostic factors for lung carcinomas. Anticancer Res. 27, 3561-3569 (2007).

33. Shaw, M. \& Cohen, P. Role of protein kinase B and the MAP kinase cascade in mediating the EGF-dependent inhibition of glycogen synthase kinase 3 in Swiss 3T3 cells. FEBS Lett. 461, 120-124 (1999).

34. Karrasch, T., Spaeth, T., Allard, B. \& Jobin, C. PI3K-dependent GSK3ss(Ser9)-phosphorylation is implicated in the intestinal epithelial cell wound-healing response. PLoS One 6, e26340 (2011).

35. Wang, H., Brown, J. \& Martin, M. Glycogen synthase kinase 3: a point of convergence for the host inflammatory response. Cytokine 53, 130-140 (2011).

36. Cross, D.A., Alessi, D.R., Cohen, P., Andjelkovich, M. \& Hemmings, B.A. Inhibition of glycogen synthase kinase-3 by insulin mediated by protein kinase B. Nature 378, 785-789 (1995).

37. Stambolic, V., Ruel, L. \& Woodgett, J.R. Lithium inhibits glycogen synthase kinase- 3 activity and mimics wingless signalling in intact cells. Curr. Biol. 6 , 1664-1668 (1996).

38. Klein, P.S. \& Melton, D.A. A molecular mechanism for the effect of lithium on development. Proc. Natl. Acad. Sci. USA 93, 8455-8459 (1996).

39. Chiu, C.T. \& Chuang, D.M. Molecular actions and therapeutic potential of lithium in preclinical and clinical studies of CNS disorders. Pharmacol. Ther. 128, 281-304 (2010).
40. Pasquali, L., Busceti, C.L., Fulceri, F., Paparelli, A. \& Fornai, F. Intracellular pathways underlying the effects of lithium. Behav. Pharmacol. 21, 473-492 (2010).

41. Afrazi, A. et al. Intracellular heat shock protein-70 negatively regulates TLR4 signaling in the newborn intestinal epithelium. J. Immunol. 188, 4543-4557 (2012).

42. Good, M. et al. Lactobacillus rhamnosus HN001 decreases the severity of necrotizing enterocolitis in neonatal mice and preterm piglets: evidence in mice for a role of TLR9. Am. J. Physiol. Gastrointest. Liver Physiol. 306, G1021-G1032 (2014).

43. Coudriet, G.M., He, J., Trucco, M., Mars, W.M. \& Piganelli, J.D. Hepatocyte growth factor modulates interleukin-6 production in bone marrow derived macrophages: implications for inflammatory mediated diseases. PLOS One 5, e15384 (2010).

44. Jope, R.S., Yuskaitis, C.J. \& Beurel, E. Glycogen synthase kinase-3 (GSK3): inflammation, diseases, and therapeutics. Neurochem. Rese. 32, 577-595 (2007).

45. Martin, M., Rehani, K., Jope, R.S. \& Michalek, S.M. Toll-like receptormediated cytokine production is differentially regulated by glycogen synthase kinase 3. Nat. Immunol. 6, 777-784 (2005).

46. Pinto, D., Gregorieff, A., Begthel, H. \& Clevers, H. Canonical Wnt signals are essential for homeostasis of the intestinal epithelium. Genes Dev. 17, 1709-1713 (2003).

47. Wahab Mohamed, W.A. \& Aseeri, A.M. Cord blood epidermal growth factor as a possible predictor of necrotizing enterocolitis in very low birth weight infants. J. Neonatal Perinatal Med. 6, 257-262 (2013).

48. Ruiz-Palacios, G.M., Cervantes, L.E., Ramos, P., Chavez-Munguia, B. \& Newburg, D.S. Campylobacter jejuni binds intestinal $\mathrm{H}(\mathrm{O})$ antigen (Fuc alpha 1, 2 Gal beta 1, 4GlcNAc), and fucosyloligosaccharides of human milk inhibit its binding and infection. J. Biol. Chem. 278, 14112-14120 (2003).

49. Newburg, D.S. Neonatal protection by an innate immune system of human milk consisting of oligosaccharides and glycans. J. Anim. Sci. 87, 26-34 (2009).

50. Feng, J. \& Besner, G.E. Heparin-binding epidermal growth factor-like growth factor promotes enterocyte migration and proliferation in neonatal rats with necrotizing enterocolitis. J. Pediatr. Surg. 42, 214-220 (2007).

51. Yu, X., Radulescu, A., Zorko, N. \& Besner, G.E. Heparin-binding EGF-like growth factor increases intestinal microvascular blood flow in necrotizing enterocolitis. Gastroenterology 137, 221-230 (2009).

52. Quaroni, A., Isselbacher, K.J. \& Ruoslahti, E. Fibronectin synthesis by epithelial crypt cells of rat small intestine. Proc. Natl. Acad. Sci. USA 75, 5548-5552 (1978) 